\title{
Non-Newtonian Blood Rheology Impacts Left Atrial Stasis in Patient-Specific Simulations
}

\author{
*A. Gonzalo ${ }^{a, b}$, M. García-Villalba ${ }^{c}$, L. Rossini $^{a}$, E. Durán ${ }^{c}$, D. Vigneault ${ }^{d}$, \\ P. Martínez-Legazpie ${ }^{e, f}$, O. Flores ${ }^{c}$, J. Bermejo ${ }^{e, f, g, h}$, E. McVeigh $^{d, i}$, A. M. Kahn ${ }^{j}$, \\ and J. C. del Alamo ${ }^{a, b, k, l}$ \\ ${ }^{a}$ Dept. of Mechanical and Aerospace Eng., University of California San Diego, La Jolla, CA, United States \\ ${ }^{b}$ Dept. of Mechanical Eng., University of Washington, Seattle, WA, United States \\ ${ }^{c}$ Dept. Bioingeniería e Ing. Aeroespacial, Universidad Carlos III de Madrid, 28911 Leganés, Spain \\ $d$ Dept. of Bioengineering, University of California San Diego, La Jolla, CA, United States \\ e Hospital General Universitario Gregorio Marañón, Madrid, Spain \\ ${ }^{f}$ Instituto de Investigación Sanitaria Gregorio Marañón, Madrid, Spain \\ $g$ Facultad de Medicina, Universidad Complutense de Madrid, Madrid, Spain \\ h CIBERCV, Madrid, Spain \\ ${ }^{i}$ Dept. of Radiology, University of California San Diego, La Jolla, CA, United States \\ $j$ Division of Cardiovascular Medicine, University of California San Diego, La Jolla, CA, United States \\ ${ }^{k}$ Center for Cardiovascular Biology, University of Washington, Seattle, WA, United States \\ ${ }^{l}$ Institute for Stem Cell and Regenerative Medicine, University of Washington, Seattle, WA, United States
}

\begin{abstract}
The lack of mechanically effective contraction of the left atrium (LA) during atrial fibrillation (AF) disturbs blood flow, increasing the risk of thrombosis and ischemic stroke. Thrombosis is most likely in the left atrial appendage (LAA), a small narrow sac where blood is prone to stagnate. Slow flow promotes the formation of erythrocyte aggregates in the LAA, also known as rouleaux, causing viscosity gradients that are usually disregarded in patient-specific simulations. To evaluate these non-Newtonian effects, we built atrial models derived from $4 \mathrm{D}$ computed tomography scans of patients and carried out computational fluid dynamics simulations using the Carreau-Yasuda constitutive relation. We examined six patients, three of whom had AF and LAA thrombosis or a history of transient ischemic attacks (TIAs). We modeled the effects of hematocrit and rouleaux formation kinetics by varying the parameterization of the CarreauYasuda relation and modulating non-Newtonian viscosity changes based on residence time. Comparing non-Newtonian and Newtonian simulations indicates that slow flow in the LAA increases blood viscosity, altering secondary swirling flows and intensifying blood stasis. While some of these effects can be subtle when examined using instantaneous metrics like shear rate or kinetic energy, they are manifest in the blood residence time, which accumulates over multiple heartbeats. Our data also reveal that LAA blood stasis worsens when hematocrit increases, offering a potential new mechanism for the clinically reported correlation between hematocrit and stroke incidence. In summary, we submit that hematocrit-dependent non-Newtonian blood rheology should be considered in calculating patient-specific indices of blood stasis indices by computational fluid dynamics.
\end{abstract}

Keywords - Computational fluid dynamics. Atrial fibrillation. Blood viscosity. Hematocrit. Thrombosis. Left atrium. 


\section{Introduction}

Ischemic stroke is a leading cause of mortality and disability. Ischemic strokes account for two thirds of all strokes, which are estimated to be 26 million per year worldwide [1]. Approximately $30 \%$ of these ischemic strokes are directly associated with atrial fibrillation (AF), a common arrhythmia affecting 35 million people worldwide [2]. An additional $30 \%$ of ischemic strokes are categorized as embolic strokes from undetermined source (ESUS), and increasing evidence suggests many of these also have a cardioembolic origin [3]. Specifically, a significant fraction of these ESUS may be linked to left atrial thrombosis, both in the presence of subclinical AF and in sinus rhythm [4]. Therefore, cardioembolism is a major source of stroke. However, the indication of anticoagulant drugs must be carefully balanced against the risk of bleeding. In patients with AF, clinical tools to estimate stroke risk are based on demographic and comorbidity factors (e.g., the $\mathrm{CHA}_{2} \mathrm{DS}_{2}$-VASc score), which do not capture patient-specific thrombogenesis mechanisms and have a modest predictive accuracy $[5,6]$. Likewise, indiscriminate anticoagulation of ESUS patients has not proven effective in preventing recurrent strokes despite increasing bleeding event rates [7]. In summary, because left atrial thrombosis is a major source of ischemic strokes, precision patient-specific indices of intracardiac thrombosis risk may improve current indications of anticoagulant therapy for stroke prevention.

Thrombogenesis is most likely with the concurrence of three phenomena: endothelial damage or dysfunction, the presence of procoagulatory factors in the blood, and increased blood stasis [8]. In the left atrium (LA), most thrombi form inside the left atrial appendage (LAA), a narrow hooked sac that accounts for approximately 5-10\% of LA volume and which varies greatly in morphology among individuals [9]. The irregular beating of the heart with a lack of organized atrial contraction, together with anatomical factors promote the appearance of LAA stasis [10, 11]. Intracavitary blood stasis and thrombogenesis risk can be quantified through blood residence time, i.e., the time span spent by blood particles inside a cardiac chamber [12], whose calculation requires detailed knowledge of the flow velocity field. Progress in computational fluid dynamics (CFD) algorithms and computer processing capacity now make it feasible to simulate blood flow in four-chamber domains [13], two-chamber domains (typically the left heart) [14, 15, 16], and single-chamber domains (typically the left ventricle) $[17,18,19]$. Some of these simulations include models of heart valve motion $[19,14,16,20]$ and some can resolve the intricate domain surface caused by endocardial trabeculation [20]. Motivated by the need for patient-specific prediction of LAA blood stasis, the number of CFD simulations of LA flow has increased rapidly in the last few years [21].

Blood inside the cardiac chambers is usually modeled as a Newtonian fluid based on the assumption that its shear rate is high enough to prevent the formation of red blood cell (RBC) aggregates and that, even if some low-shear pockets may occasionally form, their lifetime is shorter than the RBC aggregation timescale [22, 23]. While an exhaustive validation of these modeling assumptions can be challenging, Newtonian CFD simulations in chambers other than the LAA provide intracardiac flow velocity fields that agree reasonably well with phase-contrast MRI data from the same patients [18, 24]. Nevertheless, calculating residence time inside the LAA has two peculiarities that make it particularly sensitive to nonNewtonian effects. First, high-resolution CFD data suggest that both low shear rate and high residence time coexist inside the LAA [25, 26], making this site prone to RBC aggregation. Consonantly, spontaneous echocardiographic contrast or "smoke", which is caused by red blood cell aggregates under low shear [27], is frequently observed in the LAA by transesophageal echocardiography and is associated with blood stasis [28]. Second, the residence time is cumulatively integrated over multiple heartbeats in contrast to Eulerian flow metrics like kinetic energy or shear stress. Therefore, slight differences in instantaneous velocity could gradually accumulate to generate appreciable differences in residence time. Considering that blood is a shear-thinning fluid, Newtonian CFD analysis could underestimate blood stasis, and this effect could be more severe for regions of significant stasis.

The present study evaluates whether blood's shear-thinning rheology significantly affects CFD estimations of LAA blood stasis. To this end, we carried out numerical simulations of left atrial flow considering a Carreau-Yasuda constitutive relation and compared the results with those from Newtonian simulations [26]. We examined six patients covering a wide range of atrial morphologies and functions, three of whom had LAA thrombosis or a history of transient ischemic attacks (TIAs). Our results indicate that blood stasis increases in low-shear regions when non-Newtonian rheology is considered, especially in the LAA. This trend is robust for different constitutive laws that consider the timescale of RBC aggregation and the hematocrit, i.e., the volume fraction occupied by RBCs. Finally, our simulations indicate that LAA blood stasis and, by implication, LAA thrombosis risk, increase with the hematocrit level both in healthy and AF subjects. These data insinuate a novel mechanism for the clinically observed connection between high hematocrit and stroke, which had been previously attributed solely to alterations in cerebral blood flow [29]. 
bioRxiv preprint doi: https://doi.org/10.1101/2021.06.24.449801; this version posted September 9, 2021. The copyright holder for this preprint (which was not certified by peer review) is the author/funder, who has granted bioRxiv a license to display the preprint in perpetuity. It is made available under aCC-BY-NC-ND 4.0 International license.

\section{Methods}

\subsection{Patient population, image acquisition, image reconstruction, and mesh gen- eration}

We retrospectively considered the six left atrial models as in our previous work [26], consisting of 4D (3D, time-resolved) patient-specific segmentations of human left atria obtained from computed tomography (CT) imaging. Three patients had normal LA volume and function, were imaged in sinus rhythm, and did not have a left atrial appendage (LAA) thrombus. The other three patients were imaged in atrial fibrillation (AF), had an enlarged LA chamber, and impaired LA global function. Two of these subjects had an LAA thrombus which were removed digitally before running the simulations, and one had a history of transient brain ischemic attacks (TIAs). Cardiac-gated cine CT scans were performed following standard clinical protocols at each participating center (NIH, Bethesda, MD and UCSD, La Jolla, CA). The images were reconstructed using the CT scanner manufacturers' standard algorithms, yielding DICOM files with resolutions between $0.32 \mathrm{~mm}$ and $0.48 \mathrm{~mm}$ in the $x-y$ axial plane and $0.5 \mathrm{~mm}$ to $1 \mathrm{~mm}$ in the $z$-direction. Time-resolved images were obtained at regularly spaced time points across the cardiac cycle, ranging between $5 \%$ and $10 \%$ of the R-R interval.

The computational LA meshes were generated in four steps using ITK-SNAP [30] and MATLAB. The first step consisted of segmenting the 3D LA anatomy 4D CT time frame and identifying the PV inlets, mitral annulus, and LAA. For each 3D segmentation, a triangular surface mesh was created and then resampled to match the computational fluid dynamics (CFD) solver's resolution [31]. The resulting triangular meshes were registered across the cardiac cycle and their positions, yielding a coherent triangle vertex and centroid cloud [32]. Finally, the positions of these points were expressed as a Fourier temporal series to provide interpolated boundary conditions to the CFD solver at time points not contained in the 4D CT sequence. Figure 1 and Table 1 summarize the main anatomical and functional features of the patients' atria. More details on patient selection, imaging acquisition and reconstruction, and mesh generation can be found elsewhere $[26]$.

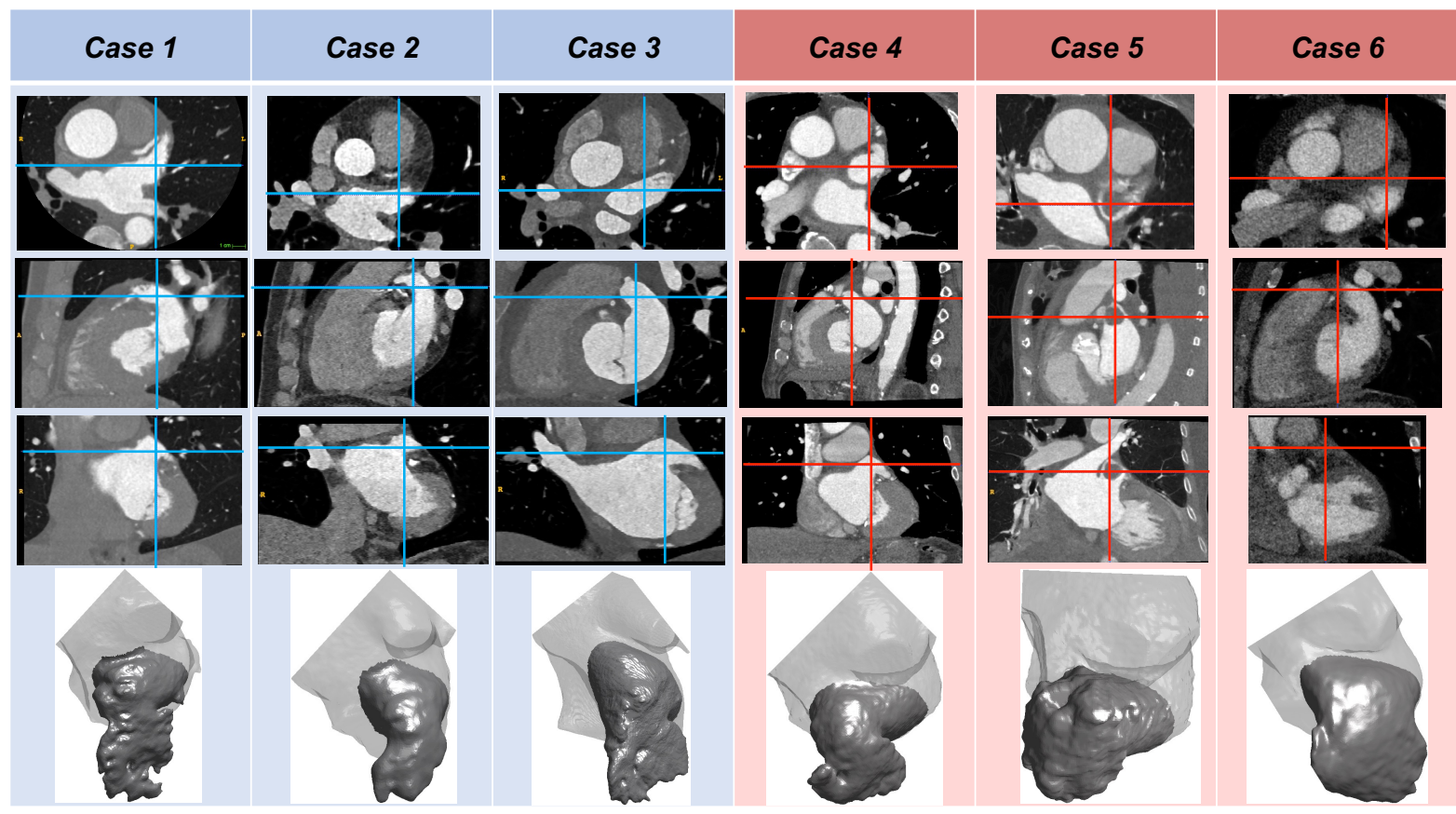

Figure 1: Anatomical Features of Patient-Specific Simulation Cases. CT images (axial, saggital, and coronal plane sections, rows 1-3 respectively) and segmented appendages (row 4) of the six left atria considered in this study. The images show LAA thrombi in cases 5 and 6 . The vertical and horizontal lines in each CT view indicate the intersections with the other two view planes. The images correspond to an instant at $50 \%$ of the R-R interval. 
bioRxiv preprint doi: https://doi.org/10.1101/2021.06.24.449801; this version posted September 9, 2021. The copyright holder for this preprint (which was not certified by peer review) is the author/funder, who has granted bioRxiv a license to display the preprint in perpetuity. It is made available under aCC-BY-NC-ND 4.0 International license.

\begin{tabular}{|c|c|c|c|c|c|c|}
\hline Subject Number & 1 & 2 & 3 & 4 & 5 & 6 \\
\hline LAA Thrombus (or history of TIA) & No & No & No & TIAs & Yes & Yes \\
\hline Sinus Rhythm & Yes & Yes & Yes & No & No & No \\
\hline Mean LA Volume (ml) & 86.6 & 70.1 & 115 & 145 & 157 & 180 \\
\hline Min. LA Volume $\left(V_{L A}^{\min }, \mathrm{ml}\right)$ & 59.6 & 49.0 & 87.2 & 119 & 150 & 157 \\
\hline Max. LA Volume $\left(V_{L A}^{\max }, \mathrm{ml}\right)$ & 108 & 91.2 & 145 & 155 & 165 & 205 \\
\hline Mean LAA Volume (ml) & 6.94 & 4.85 & 14.3 & 10.7 & 15.5 & 22.0 \\
\hline Min. LAA Volume $\left(V_{L A A}^{\min }, \mathrm{ml}\right)$ & 4.32 & 3.14 & 10.2 & 9.10 & 13.8 & 19.8 \\
\hline Max. LAA Volume $\left(V_{L A A}^{\max }, \mathrm{ml}\right)$ & 8.97 & 6.28 & 17.9 & 11.6 & 17.4 & 24.7 \\
\hline Pre-A-wave Volume $\left(V_{L A}^{p r e-A}, \mathrm{ml}\right)$ & 94.3 & 72.3 & 119 & 149 & 153 & 178 \\
\hline LA Sphericity & 0.80 & 0.82 & 0.78 & 0.86 & 0.83 & 0.81 \\
\hline Global (LA EF) $\left[\frac{V_{L A}^{\max }-V_{L A}^{\operatorname{man}}}{V_{L A}^{\max }}\right]$ & 0.45 & 0.46 & 0.40 & 0.23 & 0.091 & 0.23 \\
\hline LAA EF $\left[\frac{V_{L A A}^{\max }-V_{L A A}^{\min }}{V_{L A A}^{\max }}\right]$ & 0.52 & 0.50 & 0.43 & 0.22 & 0.21 & 0.20 \\
\hline Expansion Index $\left[\frac{V_{L A}^{\max }-V_{L A}^{\min }}{V_{L A}^{\min }}\right]$ & 0.81 & 0.86 & 0.66 & 0.30 & 0.10 & 0.31 \\
\hline Passive EF $\left[\frac{V_{L A}^{m a x}-V_{L A}^{p r a}}{V_{L A}^{m a x}}\right]$ & 0.13 & 0.21 & 0.18 & 0.039 & 0.073 & 0.13 \\
\hline Booster EF $\left[\frac{V_{L A}^{p r e-A}-V_{L A}^{\min }}{V_{L A}^{p r e-A}}\right]$ & 0.37 & 0.32 & 0.27 & 0.20 & 0.020 & 0.12 \\
\hline$\frac{\text { Conduit Volume }}{\text { LV Stroke Volume }}\left[1-\frac{V_{L A}^{\max }-V_{L A}^{\min }}{S V}\right]$ & 0.33 & 0.50 & 0.41 & 0.46 & 0.80 & 0.63 \\
\hline PVs (ml) & 43 & 38 & 51 & 36 & 17 & 49 \\
\hline PVd $(\mathrm{ml})$ & 65 & 58 & 60 & 52 & 63 & 94 \\
\hline PVs-PVd ratio & 0.66 & 0.66 & 0.84 & 0.69 & 0.27 & 0.52 \\
\hline $\mathbf{P V a}(\mathrm{ml})$ & 17 & 7.3 & 12 & 13 & 0.14 & 1.4 \\
\hline PVa duration (cycles) & 0.11 & 0.11 & 0.13 & 0.12 & 0.039 & 0.068 \\
\hline A-wave duration (cycles) & 0.22 & 0.25 & 0.30 & 0.18 & 0.21 & 0.23 \\
\hline $\mathbf{P V a}-\mathbf{A}$ wave duration ratio & 0.49 & 0.43 & 0.44 & 0.64 & 0.18 & 0.30 \\
\hline $\mathbf{E} / \mathbf{A}$ ratio & 1.64 & 2.36 & 3.03 & 2.05 & 3.86 & 3.65 \\
\hline
\end{tabular}

Table 1: Summary of anatomical and functional parameters of the left atrium (LA) and its appendage (LAA) in the LAAT/TIA-neg (left three columns) and LAAT/TIAA-pos (right three columns) groups. Mean volume indicates time-averaged volume. EF, ejection fraction; PVs, blood volume that enters the LA during LV systole; PVd, blood volume that enters the LA during LV diastole; PVa, blood volume that exits the LA due to reverse flow volume through the pulmonary veins during atrial contraction; $\mathrm{E} / \mathrm{A}$ ratio, ratio of peak mitral velocities during early diastole (E-wave) and atrial contraction (A-wave).

\subsection{Computational Fluid Dynamic}

We used a modified version of the CFD code TUCAN [33], which we had previously adapted to simulate incompressible, Newtonian flow in the left atrium [26]. To account for non-Newtonian effects, we consider the Navier-Stokes equations

$$
\frac{\partial \vec{u}}{\partial t}+(\vec{u} \cdot \nabla) \vec{u}=-\frac{1}{\rho} \nabla p+\nabla \cdot \overline{\bar{\tau}}
$$

where $\vec{u}$ and $p$ are respectively the velocity and pressure fields, $\rho$ is the fluid density, and $\tau$ is the stress tensor. The latter is defined as $\overline{\bar{\tau}}=2 \nu(S) \overline{\bar{\epsilon}}$, where $\nu$ is the fluid kinematic viscosity, $\overline{\bar{\epsilon}}=\left(\nabla \vec{u}+\nabla \vec{u}^{T}\right) / 2$ is the rate-of-strain tensor, $S=2 \sqrt{\left|\epsilon_{2}\right|}$ is the shear rate, and $\epsilon_{2}$ the second invariant of $\overline{\bar{\epsilon}}$. The constitutive relation between $\nu$ and $S$ is based on the Carreau-Yasuda model [34], both in its original form and with modifications to account for blood's thixotropic behavior under unsteady flow. Section 2.3 below provides more details about the constitutive models implemented in our CFD code.

The Navier-Stokes equations were integrated in time by a low-storage, three-stage, semi-implicit Runge-Kutta scheme using a fractional-step method. The constitutive law was treated semi-implicitly by splitting the kinematic viscosity coefficient into two terms, i.e., $\nu(S)=\nu_{i m p}+\Delta \nu_{\exp }(S)$, where $\nu_{i m p}$ is a constant coefficient. Consonantly, the viscous stresses were split as $\nabla \cdot \overline{\bar{\tau}}=\nu_{i m p} \nabla^{2} \vec{u}+\nabla \cdot\left[\Delta \nu_{\exp }(S)\left(\nabla \vec{u}+\nabla \vec{u}^{T}\right)\right]$; the first, linear term was integrated in time implicitly, whereas the second, non-linear term was integrated explicitly. We chose $\nu_{i m p}=\nu_{\infty}$ but note that the implementation is independent of this particular choice, offering some control over the stability of the temporal integration. The time step $\Delta t$ of each simulation was constant and set to keep the Courant-Friedrichs-Lewy $(C F L)$ number below 0.2 across the whole simulation. To account for the additional non-linear terms originating from $\Delta \nu_{\exp }(S)$, the Courant number was re-defined as $C F L_{\text {mod }}=\left[|\vec{u}|+\left|\nabla\left(\Delta \nu_{\text {exp }}\right)\right|\right] \Delta t / \Delta x=C F L+\left|\nabla\left(\Delta \nu_{\text {exp }}\right)\right| \Delta t / \Delta x$. The spatial discretization was performed on a staggered Cartesian grid with a centered, second-order, finite difference scheme. The grid spacing, 
bioRxiv preprint doi: https://doi.org/10.1101/2021.06.24.449801; this version posted September $9,2021$. The copyright holder for this

preprint (which was not certified by peer review) is the author/funder, who has granted bioRxiv a license to display the preprint in perpetuity. It is made available under aCC-BY-NC-ND 4.0 International license.

$\Delta x=0.51 \mathrm{~mm}\left(256^{3}\right.$ grid points), was isotropic and uniform. We initialized each non-Newtonian simulation using the velocity field previously calculated with Newtonian rheology in the same LA mesh [26]. Then, the simulations were run for 15 heartbeats in a grid of relatively coarse resolution $\left(\Delta x_{c}=16 \Delta x / 9,144^{3}\right.$ grid points) to let the flow transition from Newtonian to non-Newtonian. Finally, we interpolated the flow variables into the $256^{3}$ nominal grid and ran the simulations for 6 more heartbeats.

The time spent by blood particles inside the LA chamber was denoted residence time, $T_{R}$, and calculated together with the velocity field by solving the forced transport equation [35]

$$
\frac{\partial T_{R}}{\partial t}+\nabla \cdot\left(\vec{u} T_{R}\right)=1
$$

This equation was integrated in time explicitly with a low-storage, three-stage Runge-Kutta scheme. Its spatial discretization third-order WENO scheme to balance accuracy with preventing spurious oscillations caused by the Gibbs phenomenon in regions with sharp gradients [36]. Together with the shear rate, the residence time is used as an indicator of blood stasis and non-Newtonian rheology.

The flow was driven by the heart's wall motion obtained from patient-specific 4D CT images, which was imposed using the immersed boundary method (IBM) as previously described [26]. In short, the segmented LA surface was placed inside a $13 \mathrm{~cm}$ cubic Cartesian mesh, and free-slip boundary conditions were imposed at the mesh boundaries. No-slip boundary conditions were imposed at the LA inner surface by adding an appropriate volumetric force term to the Navier-Stokes equations. To enforce flow rates through these pulmonary vein (PV) inlets, a buffer region was defined upstream of each PV inlet plane and the IBM was applied in this region to bring the flow velocity to the desired value. A similar procedure was used to set $T_{R}$ equal to zero at the PV inlets. No additional boundary conditions are needed for the $T_{R}$ equation, which only contains first-order derivatives.

The flow velocity through each PV inlet was $\vec{v}_{i}=\frac{Q_{i}}{A_{i}} \vec{n}$, where $Q_{i}, A_{i}$ and $\vec{n}$ are the flow rate, inlet area, and normal vector $(i=1 \ldots 4)$. The $Q_{i}$ 's were determined from mass conservation as $Q_{i}=\frac{1}{4}\left(\frac{d V_{L A}}{d t}-\frac{d V_{L V}}{d t}\right)$, where $V_{L A}$ and $V_{L V}$ are the time-dependent left atrial and left ventricular volumes obtained from each patient's $4 \mathrm{D}$ CT scan. This $1 / 4$ factor evenly splits the total flow rate through the four PVs.

\section{$2.3 \quad$ Non-Newtonian constitutive models}

We ran simulations using two different rheological models. Each model was parameterized considering two hematocrit values Hct, leading to four constitutive laws in the simulations. First, we used the original Carreau-Yasuda model

$$
\nu(S)=\nu_{\infty}+\left(\nu_{0}-\nu_{\infty}\right)\left(1+(\lambda S)^{a}\right)^{\frac{n-1}{a}},
$$

where, $\lambda, n<1$, and $a$ are blood hematocrit-dependent parameters and $\nu_{\infty}$ is the reference Newtonian viscosity corresponding to the limit $S \gg \lambda^{-1}$. In this model, yield-stress is represented by prescribing a large value to the zero-shear-rate viscosity coefficient, $\nu_{0}$, which we prescribed as $\nu_{0}=16 \nu_{\infty}$ similar to De Vita et al. [37].

To investigate the effect of $H c t$, we considered two different sets of parameter values derived from the literature, namely $\lambda=8.2 \mathrm{~s}, a=0.64, n=0.2128$ from Leuprecht and Perktold [38], and $\lambda=3.313 \mathrm{~s}, a=2, n=0.3568$ from Al-Azawy et al. [39]. Although these studies considered $\nu_{\infty}=0.035 \mathrm{~cm} / \mathrm{s}^{2}$, we chose $\nu_{\infty}=0.04 \mathrm{~cm} / \mathrm{s}^{2}$ to compare with our previous Newtonian simulations [26], which where run with a constant kinematic viscosity equal to $0.04 \mathrm{~cm} / \mathrm{s}^{2}$. Figure $2 \mathrm{~A}$ shows that the shear-rate-dependent kinematic viscosities obtained from the two parameter sets differ significantly. We used the blood rheology experiments of Chien et al. [40] to estimate their associated Hct values, achieving best fits for Hct $=37.4$ and 55 (Figure 2A). Hence, we refer to these models as CY-37 and CY-55 throughout this manuscript. Blood hematocrit levels can vary significantly with age and sex, with all-age normal ranges (defined as the 2.5-97.5 percentile range) being [34-36, 48-50] for females and [39-40,54] for males [41, 42]. Thus, the CY-37 and CY-55 models are helpful to estimate the smallest and largest non-Newtonian effects to be expected in LA hemodynamics.

The Carreau-Yasuda constitutive relation assumes microstructural equilibrium but blood is a thixotropic fluid that exhibits time-dependent rheology because the formation and rupture of RBC rouleaux does not occur infinitely fast [34]. While the observation of LA and LAA echocardiographic "smoke" in patients demonstrates that rouleaux do form in these chambers [27, 28], the unsteady nature of atrial hemodynamics calls for evaluating the interplay between fluidic and thixotropic timescales. Huang and Fabisiak [43] modeled the thixotropic behavior of blood using a power-law term restrained by an exponential term that considered the cumulative exposure of RBCs to shear, i.e., proportional to $S^{m} \exp \left(-T_{1}^{-1} \int_{0}^{t} S^{m} d t\right)$. In this model, $T_{1}$ is the time scale associated to rouleaux formation and breakdown. Parameter fitting to human blood samples in healthy subjects yielded $T_{1}$ values ranging between 3.6 and 6.2 seconds. Consonantly, Schmid-Schönbein et al. [44] had previously reported the half-time for RBC aggregation in blood to be 3-5 seconds for normal blood samples and 0.5-1.5 seconds for pathologically hypercoagulable samples. More recently, Arzani [23] proposed a modified Carreau-Yasuda constitutive relation where non-Newtonian effects are activated based on the local value of the residence time. In the same spirit, we ran simulations where blood viscosity was given by

$$
\nu\left(S, T_{R}\right)=\nu_{\infty}+H\left(T_{R}\right)\left(\nu_{0}-\nu_{\infty}\right)\left(1+(\lambda S)^{a}\right)^{\frac{n-1}{a}},
$$



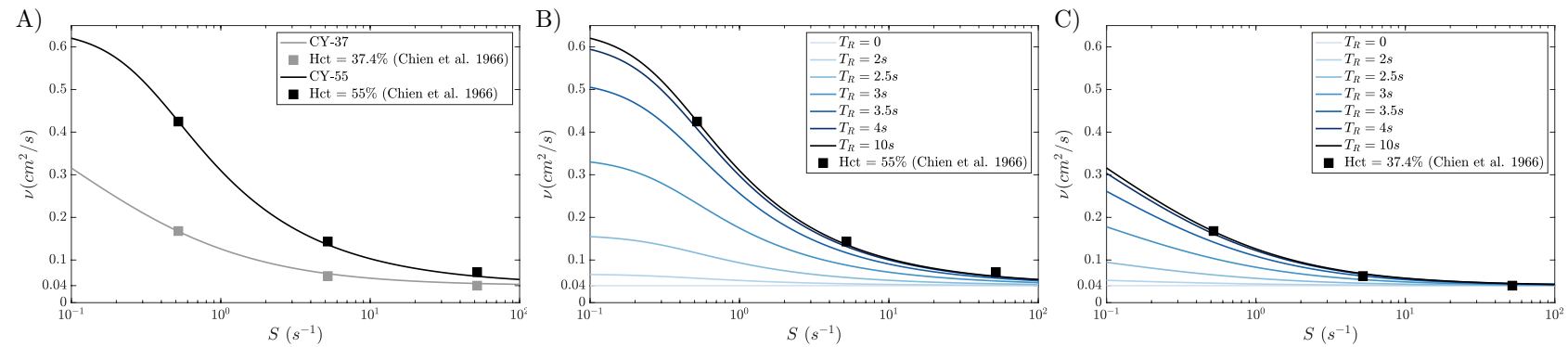

Figure 2: Non-Newtonian constitutive laws used in the present simulations. A) Kinematic viscosity $\nu(S)$ as a function of shear rate $S$ for the two versions of the Carreau-Yasuda law (eq. 3) used in our simulations. Grey line: CY-37 model with $\lambda=8.2 \mathrm{~s}, a=0.64, n=0.2128, \nu_{0}=16 \nu_{\infty}$, and $\nu_{\infty}=0.04 \mathrm{~cm} / \mathrm{s}^{2}$; black line: CY-55 model with $\lambda=3.313 \mathrm{~s}, a=2, n=0.3568, \nu_{0}=16 \nu_{\infty}$, and $\nu_{\infty}=0.04 \mathrm{~cm} / \mathrm{s}^{2}$. The square symbols correspond to datapoints from the empirical law of Chien et al. [40] for hematocrit values $H c t=37.4 \%$ (grey) and $H c t=55 \%$ (black). Kinematic viscosity $\nu\left(S, T_{R}\right)$ as a function of shear rate $S$ and residence time $T_{R}$ for the residence-time-activated Carreau-Yasuda models B) CY- $T_{R}-55$ and C) CY- $T_{R}-37$ (eqs. 4-5). Black squares come from Chien et al.'s [40] data for the respective Hct values.

$$
H(T)=\frac{1+\operatorname{erf}\left[\left(T-T_{\mu}\right) /(\sqrt{2} \sigma)\right]}{2}, T_{\mu}=3 \mathrm{~s}, \sigma=0.6 \mathrm{~s}
$$

which provides a smooth transition from Newtonian to non-Newtonian behavior for residence times comparable to experimentally reported RBC aggregation timescales $[44,43]$. We ran simulations at $H c t=37$ and 55 with the constitutive model in eqs. (4-5), which we denoted as CY- $T_{R}-37$ and CY- $T_{R}-55$. Figure $2 \mathrm{~B}$ illustrates the dependence of the CY$T_{R}-55$ model with residence time, showing that non-Newtonian effects are negligible for $T_{R} \lesssim 2 \mathrm{~s}$, gradually increase for $2 \mathrm{~s} \lesssim T_{R} \lesssim 10 \mathrm{~s}$, and become indistinguishable from those of the classic Carreau-Yasuda model for $T_{R} \gtrsim 10 \mathrm{~s}$. The dependence of the CY- $T_{R}-37$ model with residence time is similar (Figure $2 \mathrm{C}$ ).

\section{Results}

\subsection{Flow visualization in Newtonian and non-Newtonian left atrial simulations}

We first evaluated whether the LA body and LAA flow patterns were affected by non-Newtonian blood rheology. Figure 3 displays 3D maps of blood velocity vectors in a representative normal subject (case 2), computed by the Newtonian simulations as well as the non-Newtonian CY-37, and CY-55 simulations. The vectors near the left PVs, the right PVs, the LAA, and the mitral annulus are colored differently to facilitate visualization. Magnified 3D vector maps inside the LAA are also displayed. Snapshots corresponding to LA diastole, LV early filling, and LA systole are represented. For reference, the figure includes flow rate profiles of the pulmonary veins (PVs) and mitral annulus, together with LA and LAA chamber volume vs. time. Figure 4 shows similar simulation results for a representative AF, LAA-thrombus-positive patient (case 5).

For the most part, the flow patterns inside the LA body were unaltered in the non-Newtonian CY-37 and CY-55 simulations. In the healthy cases, atrial chamber expansion during atrial diastole drove a strong flow from the PVs to the mitral annulus (Figure 3A), which was sustained by ventricular suction and elastic atrial recoil during LV filling (Figure 3B). Atrial contraction (Figure 3C) kept squeezing blood out of the LAA and the LA body into the ventricle, also creating backflow from the LA into the PVs. Non-Newtonian effects did not seem to significantly alter the flow patterns in the LA body of AF patients either. The LA mainly acted as a conduit with strong fluid motion from the PVs into the ventricle during LV early filling (Figure 4B), while the flow was practically arrested during the remainder of the cardiac cycle (Figure 4A, C).

On the other hand, we found appreciable qualitative and quantitative differences in flow patterns inside the LAA. First, the filling and drainage LAA jets were altered when non-Newtonian effects were considered. In the healthy subject, these differences were most noticeable during LV relaxation and atrial systole (Figure 3B-C). In the LAA-thrombus-positive $\mathrm{AF}$ patient, the differences were sustained throughout the cardiac cycle (Figure 4A-C). Moreover, the AF patients experienced considerable secondary swirling flows in their LAAs, which were significantly more voluminous and had less straight shapes than the healthy ones (e.g., $15.5 \mathrm{ml}$ vs. $4.85 \mathrm{ml}$ for the cases shown in Figures $3-4$, see Table 1). These swirling motions showed significant alterations in the non-Newtonian simulations, including an important decrease of velocity magnitude (Figure 4). 
bioRxiv preprint doi: https://doi.org/10.1101/2021.06.24.449801; this version posted September 9, 2021. The copyright holder for this
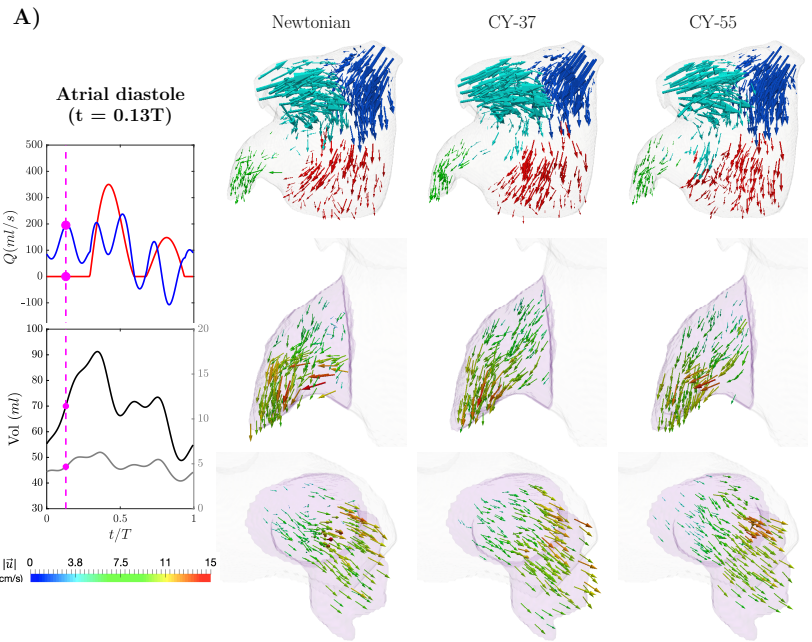

B)
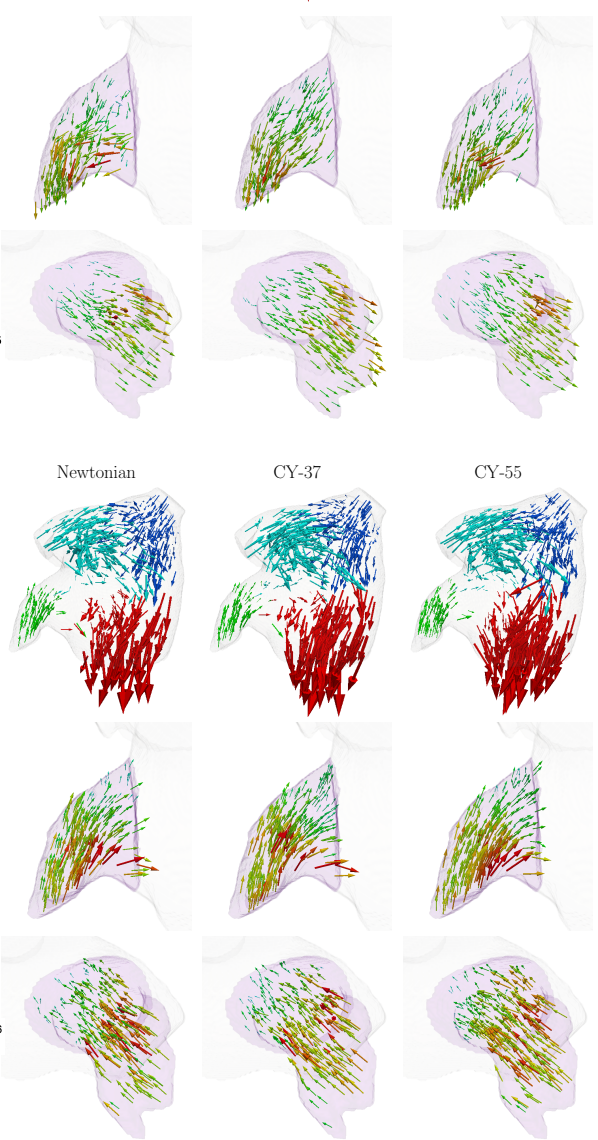

C)
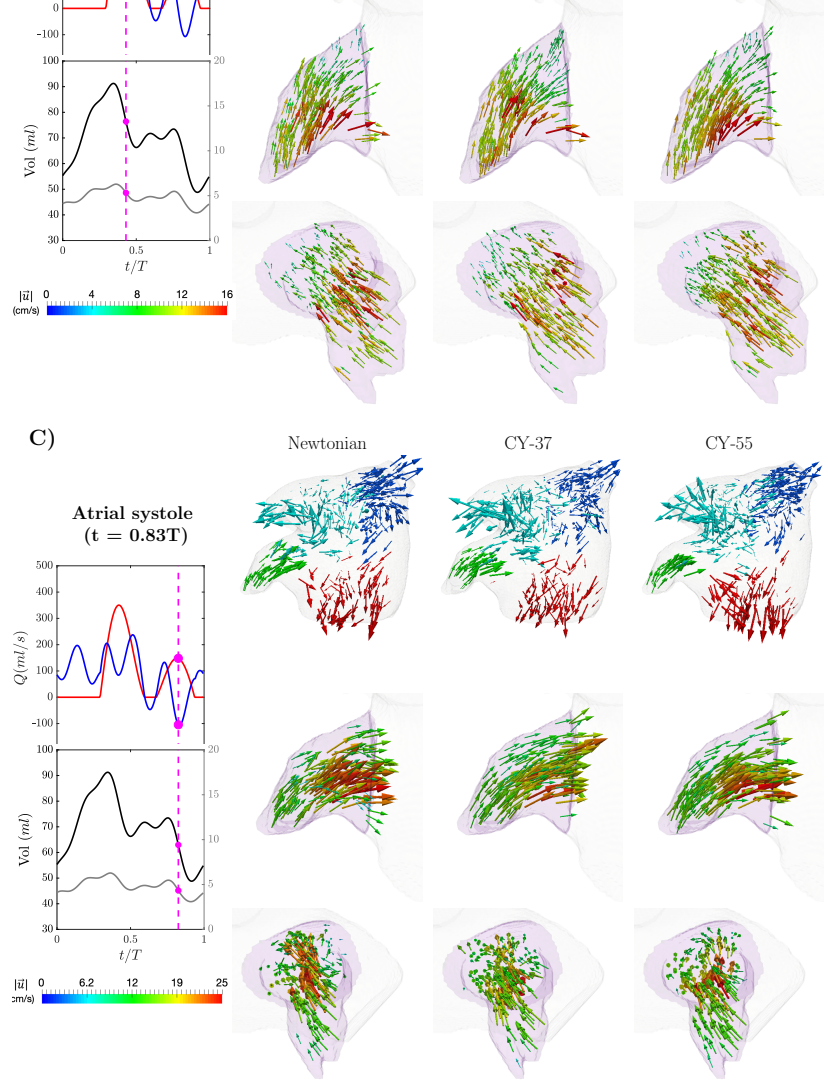

Figure 3: Flow visualization of LA and LAA hemodynamics from Newtonian and non-Newtonian simulations. Subject with normal atrial function and no LAA thrombus. Each panel displays a snapshot of the 3-D blood flow velocity in the whole LA (top) and two amplified views of the LAA in different orientations. In the top view, the vectors are colored according to their proximity to the right pulmonary veins (blue), left pulmonary veins (cyan), left atrial appendage (green) and mitral valve (red). In the bottom LAA views, the vectors are colored according to the velocity magnitude. The vectors in all views are scaled with the velocity magnitude. Each panel also includes time histories of the flow rate through the mitral valve (red) and the cumulative flow rate through the pulmonary veins (blue), and of the volumes of the left atrium (black) and the left atrial appendage (grey). The magenta bullets indicate the instant of time represented in the vector plots of each panel. The flow vectors inside the LA are represented at three instants of time. A) Atrial diastole and peak flow rate through the pulmonary veins $(t=0.13 \mathrm{~s})$. B) Left ventricular diastole and peak flow rate through the mitral valve $(\mathrm{E}$-wave, $\mathrm{t}=0.43 \mathrm{~s})$. C) Atrial systole and peak backflow rate through the pulmonary veins $(t=0.83 \mathrm{~s})$. 
bioRxiv preprint doi: https://doi.org/10.1101/2021.06.24.449801; this version posted September 9, 2021. The copyright holder for this

A)
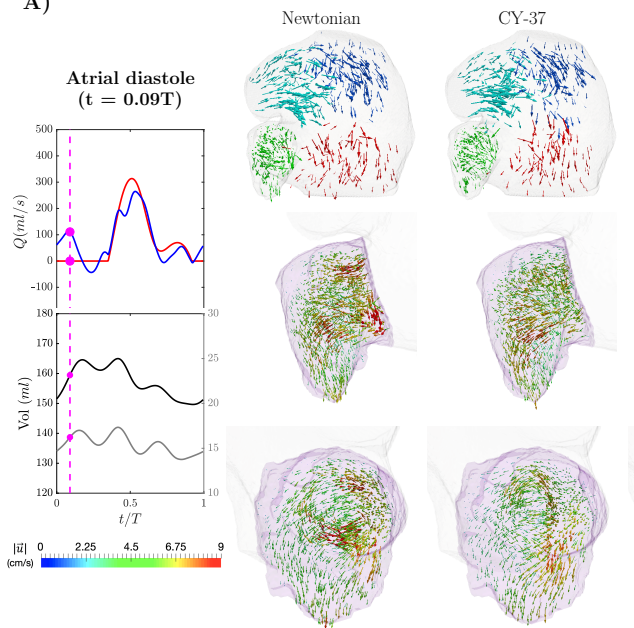

B)
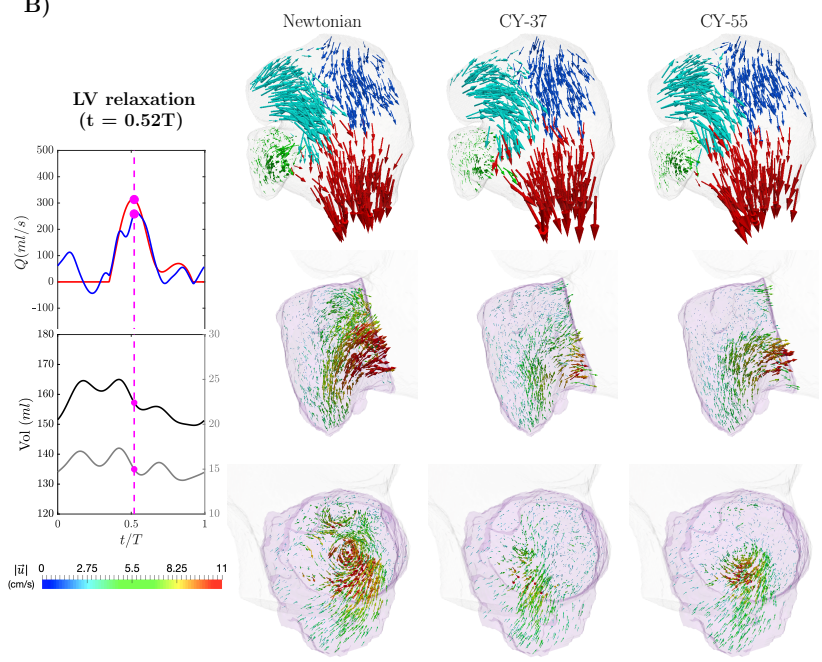

C)
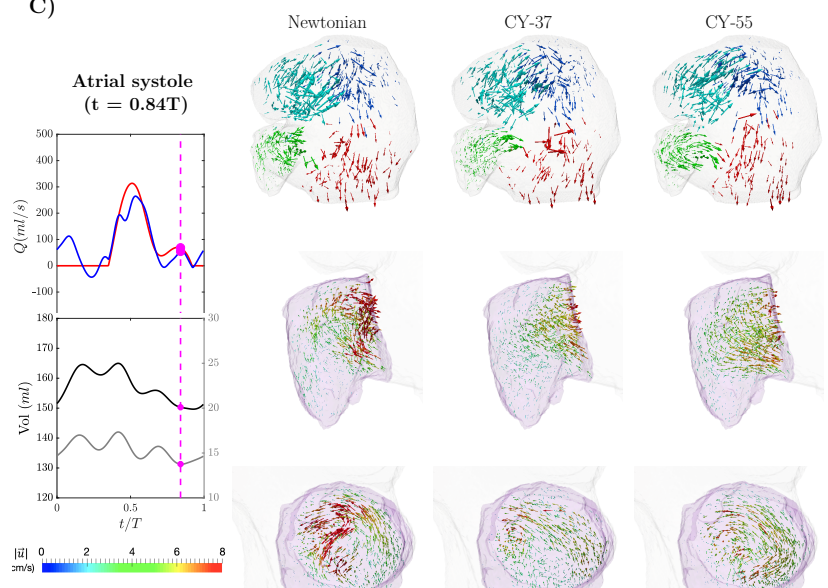
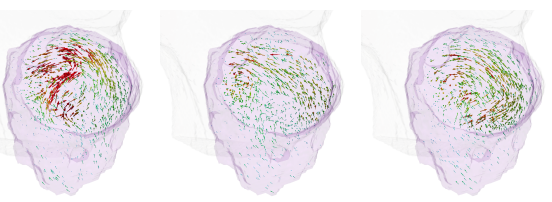

Figure 4: Flow visualization of LA and LAA hemodynamics from Newtonian and non-Newtonian simulations. AF patient with LAA thrombus (digitally removed before running the simulations). Vector maps of the 3-D blood flow velocity in the whole LA (top) and two amplified views of the LAA in different orientations, using the same format as Figure 3. A) Atrial diastole and peak flow rate through the pulmonary veins $(\mathrm{t}=0.09 \mathrm{~s})$. B) Left ventricular diastole and peak flow rate through the mitral valve $(\mathrm{E}$-wave, $\mathrm{t}=0.52 \mathrm{~s})$. C) Atrial systole and peak backflow rate through the pulmonary veins $(t=0.84 \mathrm{~s})$. 


\subsection{Blood inside the left atrial appendage continuously experiences low shear rates consistent with non-Newtonian rheology}

Since low shear rates and RBC aggregation are hallmarks of non-Newtonian blood rheology, we analyzed the shear rate $S(\vec{x}, t)$ in our simulations. Figure 5 shows instantaneous snapshots of $S(\vec{x}, t)$ from Newtonian and CY-37 simulations. The data come from normal case 3, an AF patient with decreased LA reservoir function (case 4, history of TIAs), and an $\mathrm{AF}$ patient with decreased reservoir and booster functions (case 5, thrombus positive). To visualize LA vortices in the atrial body, we also represented iso-surfaces of the second invariant of the velocity gradient tensor $\left(Q=1000 \mathrm{~s}^{-2}\right)$.

Given the relatively large Reynolds number and the unsteady nature of LA flow, the shear rate maps fluctuate in space and time. However, despite these fluctuations, $S(\vec{x}, t)$ remained low $\left(<10 \mathrm{~s}^{-1}\right)$ in large portions of the LAA across the whole cardiac cycle. Since the CY-37 and CY-55 constitutive models yield $\nu / \nu_{\infty}=1.3$ and 2 respectively at $S=10 \mathrm{~s}^{-1}$, the $S(\vec{x}, t)$ distributions from our simulations suggest that non-Newtonian effects could be considerable inside the LAA. The subjects with decreased atrial function had particularly low shear rates inside their LAA. These subjects also displayed low $S$ regions inside the atrial body, but these regions were not sustained over long times. The distributions of $S(\vec{x}, t)$ for the Newtonian and CY-37 blood simulations were similar in that they produced low shear rates inside the LAA across the whole cardiac cycle. The LA vortex structures were qualitatively similar, although there was a trend for non-Newtonian simulations to yield increased velocities along intense vortex cores, which is to be expected in shear-thinning fluids. 
bioRxiv preprint doi: https://doi.org/10.1101/2021.06.24.449801; this version posted September 9, 2021. The copyright holder for this preprint (which was not certified by peer review) is the author/funder, who has granted bioRxiv a license to display the preprint in perpetuity. It is made available under aCC-BY-NC-ND 4.0 International license.

A) Case 3. Normal LA Function. Thrombus negative


B) Case 4. Impaired Reservoir Function. Thrombus negative (history of TIAs)
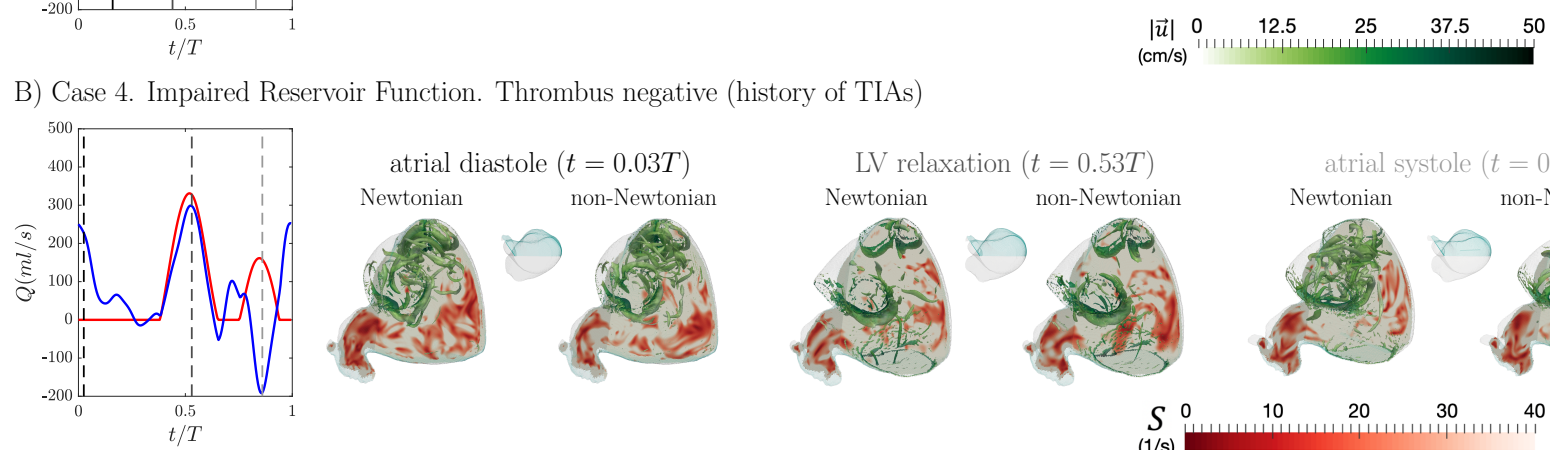

C) Case 5. Impaired Reservoir and Booster Function. Thrombus positive
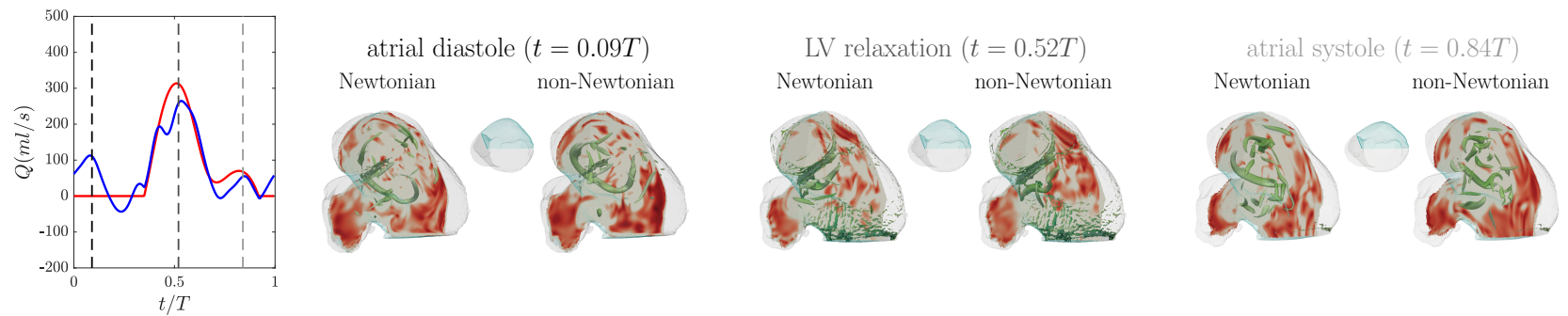

Figure 5: Left atrial shear rate and vortex structures inside left atrium of subjects with or without LAA thrombus and with different atrial function. Three subjects are shown, one without thrombus and normal atrial function (panel A), one with a history of TIAs and impaired reservoir function (panel B), and one with an LAA thrombus and both impaired reservoir and booster functions (panel $\mathbf{C}$ ). Each panel includes plots of the time histories of flow rate through the mitral valve (red) and the cumulative flow rate through the PVs (blue). For each subject, instantaneous snapshots of the shear rate $S$ are shown in two oblique plane sections of the LAA and the LA body, indicated the sagittal view insets. Additionally, the vortex patterns inside the LA are represented and colored according to the local velocity magnitude. Snapshots from both Newtonian and CY-37 simulations (Carreau-Yasuda model of eq. 3, Hct =37) are included. These snapshots correspond to three instants of time: 1) atrial diastole (i.e., peak flow rate through the pulmonary veins), 2) left ventricular rapid filling (i.e., the E-wave, peak flow rate through the mitral valve), and 3) atrial systole (i.e., the A-wave of left ventricular filling and peak backflow rate through the pulmonary veins). These three instants of time are indicated with dashed vertical lines in the flow-rate plots at the left-hand-side of each panel.

\subsection{Blood viscosity in Newtonian and non-Newtonian left atrial simulations}

To evaluate the variations in atrial blood viscosity caused by the shear rate dependence and thixotropy of blood rheology, we determined the normalized time-averaged kinematic viscosity over the last three heartbeats of our simulations, $\langle\nu(\vec{x})\rangle / \nu_{\infty}$. We mapped this quantity in two oblique intersecting plane sections of the LA body and the LAA for the same two cases considered in Figures 3 and 4 (Figure 6A). In addition, we plotted time histories of the median of $\nu / \nu_{\infty}$ inside the LA body and LAA (Figure 6B), and the probability density functions of $\nu / \nu_{\infty}$ inside the LAA for the last simulated cycle (Figure 6C). Consistent with the sustained presence of low-shear regions inside the left atrium, Figure 6 indicates that the CY-37 and CY-55 simulations yielded higher viscosity than the Newtonian simulation. This increase was particularly appreciable inside the LAA.

Table 2 displays statistics of non-Newtonian effects for all our simulations. The low-hematocrit CY-37 simulations produced moderate increases in viscosity for healthy subjects (range 1.18-1.25), which were mostly confined inside the LAA. There was a trend for the CY-37 simulations to produce higher increases in viscosity in AF patients (range 1.31-1.38) than in healthy ones, with more extensive zones of elevated $\langle\nu(\vec{x})\rangle / \nu_{\infty}$ inside the LAA and and the LA body. The high-hematocrit CY-55 simulations produced significantly larger viscosity values all over the LA body and LAA, with $\langle\nu(\vec{x})\rangle / \nu_{\infty}=1.99$ and 2.56 for the healthy and AF patients. 
bioRxiv preprint doi: https://doi.org/10.1101/2021.06.24.449801; this version posted September 9, 2021. The copyright holder for this preprint (which was not certified by peer review) is the author/funder, who has granted bioRxiv a license to display the preprint in perpetuity. It is made available under aCC-BY-NC-ND 4.0 International license.

\begin{tabular}{|c|c|c|c|c|c|c|c|c|c|c|c|c|c|c|c|c|c|}
\hline Viscosity model & $v_{0}$ & $v_{\infty}$ & Hct & $\lambda$ & a & $\mathrm{n}$ & $\mid T_{\mu}(s)$ & $\sigma(s)$ & Cases & $\langle S(\vec{x})\rangle\left(s^{-1}\right)$ & $\langle v(\vec{x})\rangle / v_{\infty}$ & $\mid\left\langle T_{R}(\vec{x})\right\rangle(s)$ & $\frac{\left\langle T_{R}^{N N}(\vec{x})\right\rangle-\left\langle T_{R}^{N}(\vec{x})\right\rangle}{\left\langle T_{R}^{N}(\vec{x})\right\rangle}(\%)$ & $\left\langle T_{R, \max }(\vec{x})\right\rangle(s)$ & $\% \mathrm{Vol} T_{R}>6 s$ & $\%$ Voll $T_{R}>8 s$ & $\% \mathrm{Vol} T_{R}>10 \mathrm{~s}$ \\
\hline Newtonian & \multirow{9}{*}{0.64} & \multirow{9}{*}{0.04} & - & - & - & - & $\overline{c-}$ & - & 1 & 23.85 & 1.00 & 2.27 & - & 8.83 & 8.29 & 0.54 & 0.00 \\
\hline $\mathrm{CY}$ & & & 37 & 8.2 & 0.64 & 0.2128 & - & - & 1 & 22.93 & 1.18 & 2.87 & 26.91 & 13.63 & 28.61 & 12.46 & 3.53 \\
\hline Newtonian & & & - & - & - & - & - & - & 2 & 21.04 & 1.00 & 1.63 & - & 7.04 & 1.39 & 0.00 & 0.00 \\
\hline $\mathrm{CY}$ & & & 37 & 8.2 & 0.64 & 0.2128 & - & - & 2 & 17.87 & 1.24 & 1.99 & 22.63 & 9.71 & 9.83 & 2.27 & 0.24 \\
\hline $\mathrm{CY}$ & & & 55 & 3.313 & 2 & \begin{tabular}{|l|}
0.3568 \\
\end{tabular} & - & - & 2 & 16.97 & 1.99 & 2.12 & 30.54 & 11.37 & 19.49 & 8.81 & 1.97 \\
\hline $\mathrm{CY}-T_{R}$ & & & 37 & 8.2 & 0.64 & 0.2128 & 3 & 0.6 & 2 & 18.98 & 1.04 & 1.83 & 12.29 & 8.94 & 3.22 & 0.41 & 0.00 \\
\hline$C Y-T_{R}$ & & & 55 & 3.313 & 2 & 0.3568 & 3 & 0.6 & 2 & 18.89 & 1.15 & 2.12 & 30.54 & 8.98 & 5.41 & 0.63 & 0.00 \\
\hline Newtonian & & & - & - & - & - & - & - & 3 & 17.42 & 1.00 & 2.30 & - & 9.59 & 13.95 & 4.48 & 0.00 \\
\hline $\mathrm{CY}$ & & & 37 & 8.2 & 0.64 & 0.2128 & - & - & 3 & 17.87 & 1.25 & 3.06 & 33.07 & 12.93 & 33.39 & 22.84 & 12.06 \\
\hline Newtonian & \multirow{9}{*}{0.64} & \multirow{9}{*}{0.04} & - & - & - & - & - & - & 4 & 11.03 & 1.00 & 3.25 & - & 11.53 & 34.35 & 16.45 & 5.45 \\
\hline $\mathrm{CY}$ & & & 37 & 8.2 & 0.64 & 0.2128 & - & - & 4 & 11.66 & 1.31 & 4.30 & 32.59 & 17.83 & 40.58 & 32.42 & 24.26 \\
\hline Newtonian & & & - & - & - & - & - & - & 5 & 9.73 & 1.00 & 3.88 & - & 10.88 & 25.58 & 12.50 & 2.37 \\
\hline $\mathrm{CY}$ & & & 37 & 8.2 & 0.64 & 0.2128 & - & - & 5 & 8.29 & 1.38 & 4.01 & 3.23 & 16.06 & 26.40 & 13.62 & 7.25 \\
\hline $\mathrm{CY}$ & & & 55 & & 2 & 0.3568 & - & - & 5 & 7.33 & 2.56 & 4.65 & 19.72 & 17.44 & 34.58 & 23.43 & 17.44 \\
\hline $\mathrm{CY}-T_{R}$ & & & 37 & 8.2 & 0.64 & 0.2128 & 3 & 0.6 & 5 & 7.52 & 1.27 & 4.00 & 3.06 & 18.03 & 21.06 & 12.47 & 7.01 \\
\hline $\mathrm{CY}-T_{R}$ & & & \begin{tabular}{|l|}
55 \\
\end{tabular} & 3.313 & 2 & \begin{tabular}{|l|}
0.3568 \\
\end{tabular} & 3 & 0.6 & 5 & 7.87 & 1.92 & 4.45 & 14.79 & 16.48 & 38.49 & 25.59 & 14.19 \\
\hline Newtonian & & & - & - & - & - & - & - & 6 & 12.16 & 1.00 & 2.53 & - & 7.76 & 4.84 & 0.00 & 0.00 \\
\hline $\mathrm{CY}$ & & & 37 & 8.2 & 0.64 & 0.2128 & - & - & 6 & 12.09 & 1.34 & 2.55 & 0.71 & 8.94 & 8.85 & 0.57 & 0.00 \\
\hline
\end{tabular}

Table 2: Summary of blood rheology models and cases studied, including time-averaged hemodynamical statistics evaluated in the left atrial appendage (LAA) and the distal LAA. Hct, hematocrit level; Carreau-Yasuda model parameters $\left(\nu_{0}, \nu_{\infty}, \lambda, a\right.$ and $\left.n\right)$, and modified Carreau-Yasuda model additional parameters $\left(T_{\mu}\right.$ and $\left.\sigma\right)$, see section 2.3 for description; $\% \operatorname{Vol} T_{R}>\tau$, percentage of volume with residence time larger than the time threshold $\tau$. Metrics between \langle\rangle are time averages over the phase-averaged cardiac cycle obtained in the three cycles highlighted with a grey shaded region in Figures 6 and 7.

Previous Newtonian CFD analyses (reviewed in [21]) and our own [26] suggest that LA blood residence time can be comparable to the timescale associated with blood's thixotropic behavior [44, 43]. Thus, the CY-55 and CY-37 models, which assume steady-state blood rheology, could overestimate blood viscosity in low residence time zones. To investigate how the competition between RBC aggregation kinetics and flow unsteadiness influences the results of the CFD analysis, we ran the two patient-specific cases of Figures 3 and 4 using the low CY- $T_{R}-37$ and high-hematocrit CY- $T_{R}-55$ constitutive laws (eqs. 4-5). In these thixotropic constitutive relations, non-Newtonian effects only kick in if the local residence time is longer than the activation time $T_{\mu}=3 \mathrm{~s}$. Consistent with this $T_{R}$-dependent activation, the CY- $T_{R}-55$ model yielded viscosities that were very close to the constant Newtonian reference value inside the whole LA body and in the proximal part of the LAA (Figure 6). In contrast, the CY-55 model produced significant non-Newtonian effects across the whole LA body, with $\langle\nu(\vec{x})\rangle / \nu_{\infty}=1.69-2.44$. The same trend was observed when comparing the CY- $T_{R}-37$ and CY-37 simulations, although including $T_{R}$-dependent activation had less dramatic results at $H c t=37$ than at $H c t=55$ because non-Newtonian effects were moderate at low hematocrit. The next section characterizes how the viscosity variations caused by the constitutive laws we investigated (i.e. CY-37, CY-55, CY- $T_{R}-37$, and CY- $T_{R}-55$ ) affect the predictions of blood stasis from the CFD analysis.

\subsection{Blood stasis in Newtonian and non-Newtonian left atrial simulations}

We examined how non-Newtonian blood rheology affects LAA blood stasis by mapping the blood residence time obtained with the CY-37, CY-55 constitutive laws, and comparing it to previous results reported using the constant viscosity assumption [26]. The $T_{R}$ maps indicate that most of the LA body's blood pool is cleared and replenished every 1-2 cardiac cycles $\left(T_{R} \leq 2 \mathrm{~s}\right.$, grey shades in Figure 7A). On the other hand, the LAA residence time can reach large values, particularly in the LAA regions most distal from the ostium (Figure 7A). The increase in residence time between the LA body and the appendage was larger in patients with impaired atrial function (e.g., case 5 in Figure 7), whose LAA contracted and expanded weaklier than in healthy subjects (see LA functional parameters in Table 1). The LAA was also the site where the residence time maps produced by non-Newtonian simulations differed most from the Newtonian ones. These results are consistent with our findings that non-Newtonian effects are most significant inside the LAA (Figure 6) and that these effects weaken the filling, drainage, and stirring of blood inside this chamber (Figures 3 and 4). The CY-37 and CY-55 simulations exacerbated high- $T_{R}$ stagnant regions by augmenting their size and maximum residence time. Conversely, regions of moderate or low $T_{R}$ were not affected as much. These differences were significant even in the low hematocrit simulation, CY-37, and became more pronounced in the high hematocrit case, CY-55. For instance, the apical LAA residence times predicted by the CY-37 simulation for the healthy and AF cases of Figure 7 were $38 \%$ and $47 \%$ higher than their Newtonian values, respectively, whereas these differences were $62 \%$ and $60 \%$ when considering the CY-55 constitutive law. 
bioRxiv preprint doi: https://doi.org/10.1101/2021.06.24.449801; this version posted September 9, 2021. The copyright holder for this

A)
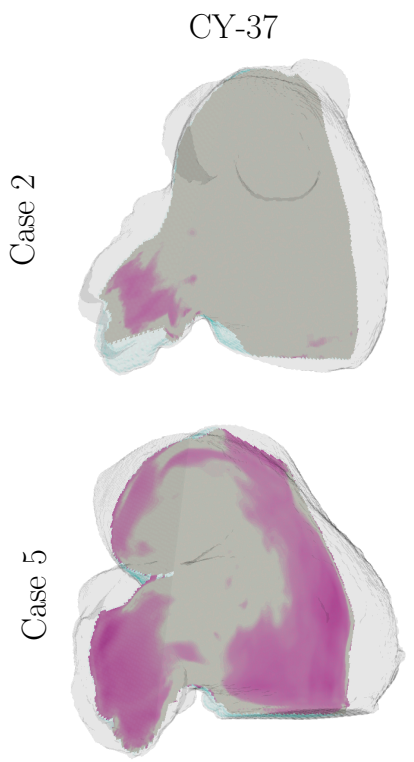

B)

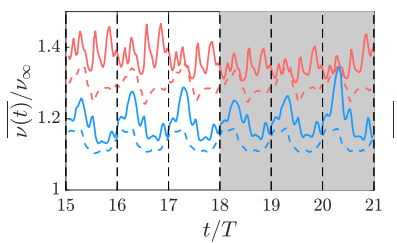

C)
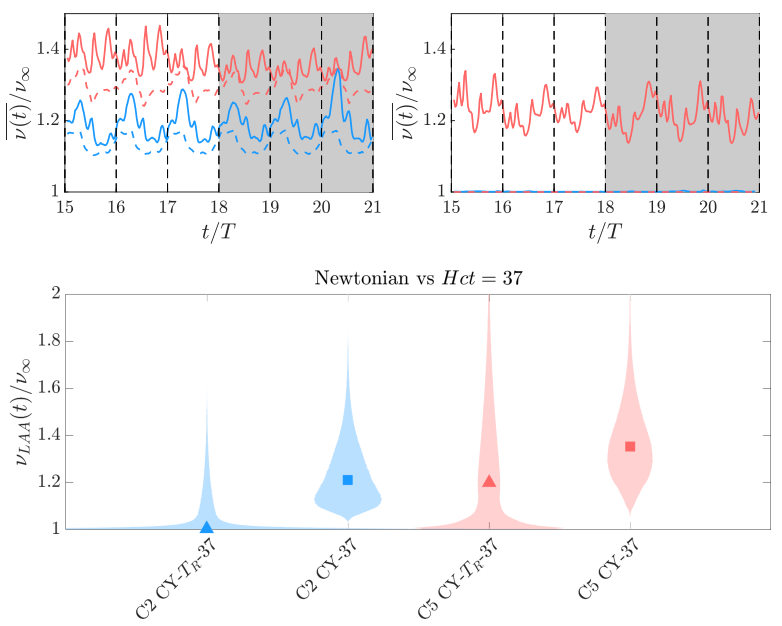

CY-55
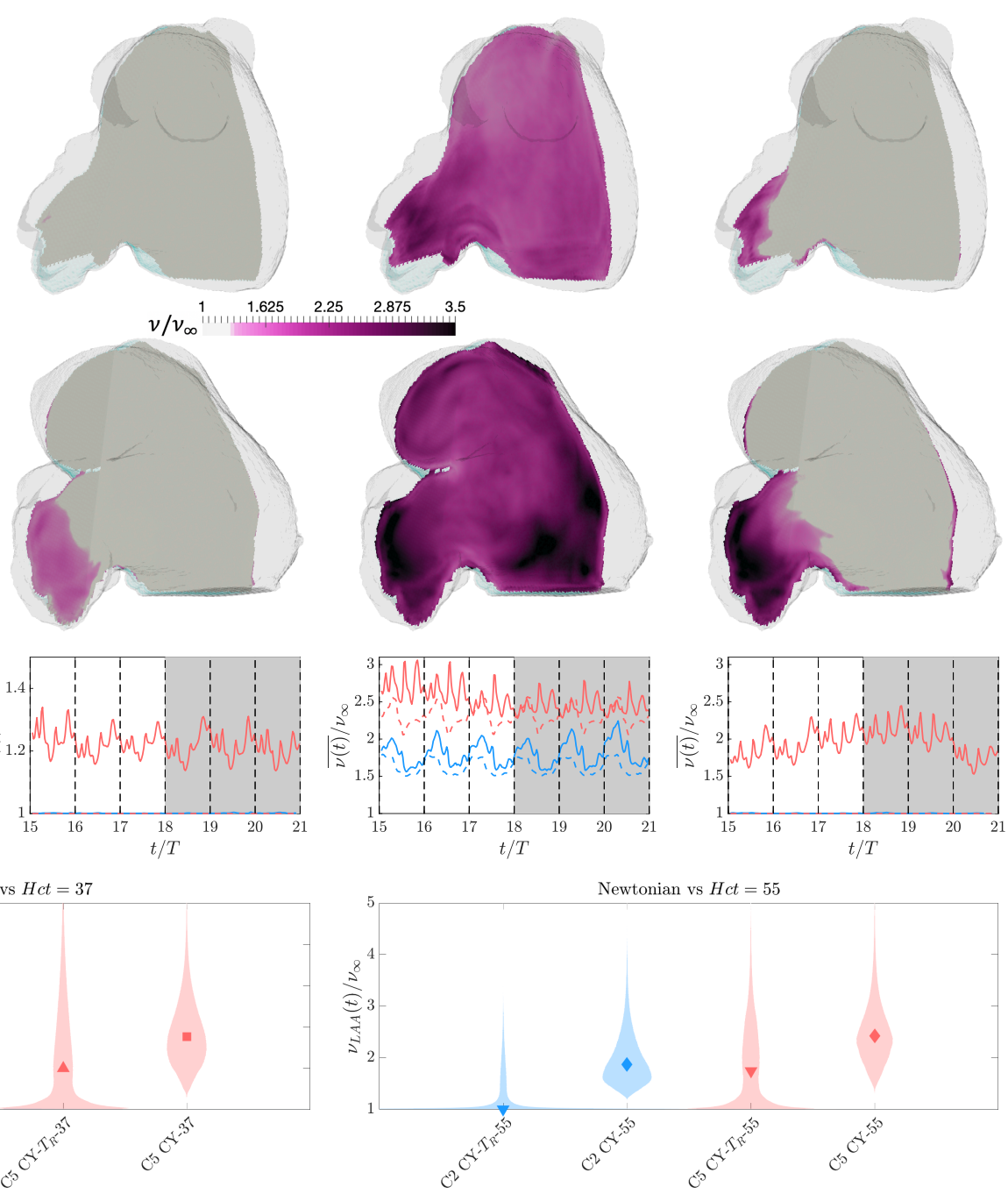

Figure 6: Left atrial viscosity for non-Newtonian different constitutive laws. A) Spatial distribution of kinematic viscosity. Two subjects are shown, one without thrombus and normal atrial function (top), and one with an LAA thrombus (digitally removed before running the simulations) and both impaired reservoir and booster functions (bottom). For each subject, the time-averaged, normalized viscosity $\langle\nu(\vec{x})\rangle / \nu_{\infty}$ is shown in two oblique plane sections of the LAA and the LA body, similar to Figure 5. Data from simulations with low-hematocrit CY-37 ( $1^{\text {st }}$ column), low-hematocrit, residence-time-activated CY- $T_{R^{-}} 37$ ( $2^{\text {nd }}$ column), high-hematocrit CY-55 ( $3^{\text {rd }}$ column), and high-hematocrit, residence-time-activated CY- $T_{R^{-}} 55$ ( $4^{\text {th }}$ column) constitutive laws are included. B) Time histories of kinematic viscosity from the healthy, thrombus-negative subject (blue) and the thrombus-positive patient (red) with AF shown in panel A. Data from the last 6 cycles of the simulations are plotted, with the grey shaded region indicating the three cycles used to calculate time averages (i.e., $\langle\nu(\vec{x})\rangle / \nu_{\infty}$ in panel A and panel C). Lines represent the median. Solid lines indicate the LAA while dashed lines indicate the LA body. C) Violin plots of the probability density function (shaded patches) inside the LAA, together with the median (symbol). Colors as in panel B. 
bioRxiv preprint doi: https://doi.org/10.1101/2021.06.24.449801; this version posted September 9,2021 . The copyright holder for this

A)
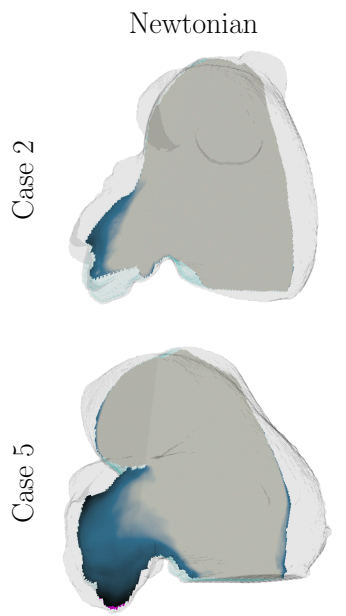

B)

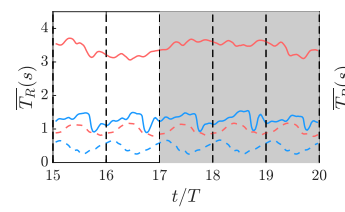

CY-37
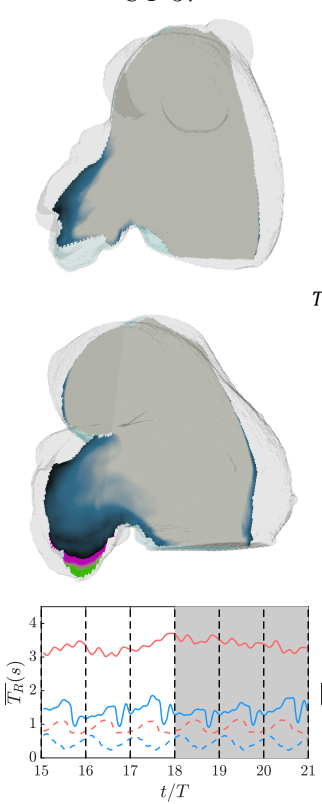

CY-T $R^{-37}$
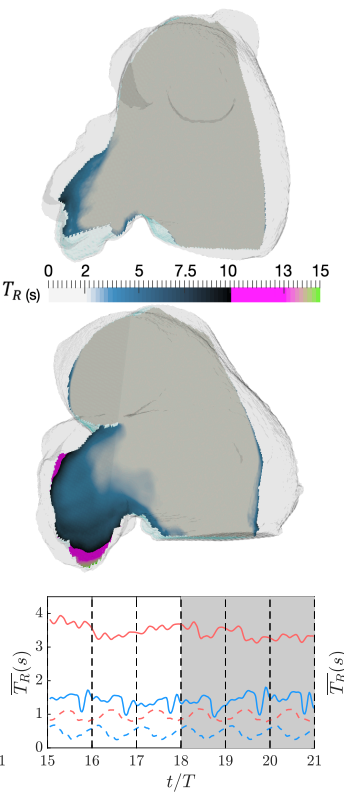

CY-55
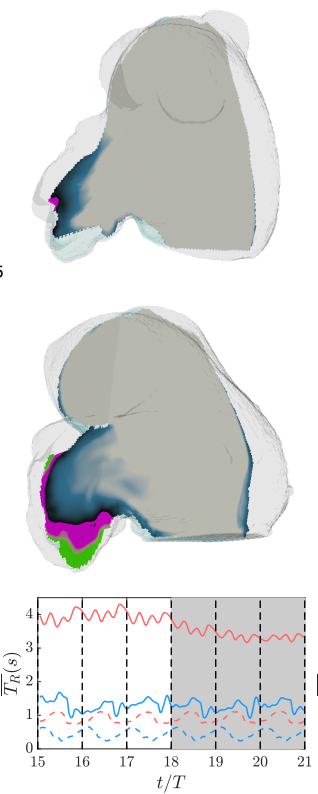

CY- $T_{R^{-}} 55$
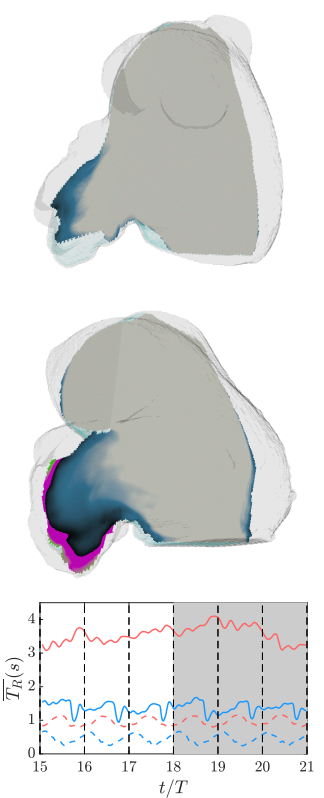

C)
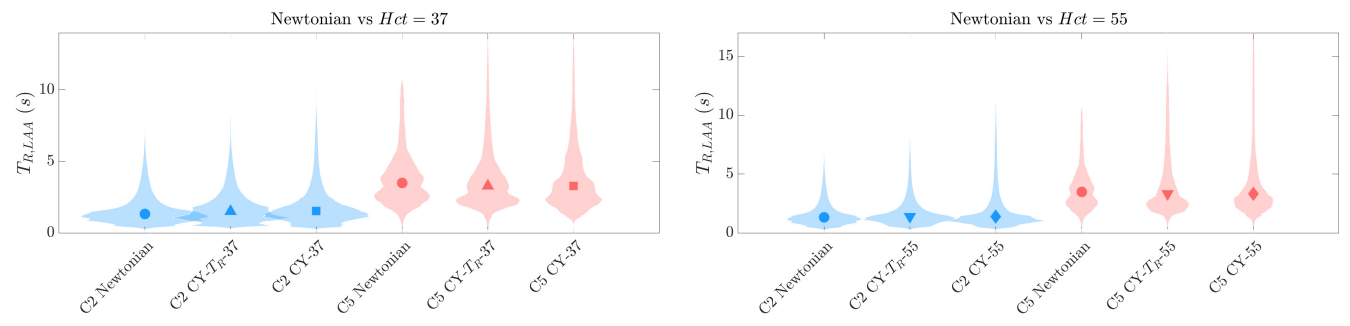

Figure 7: Left atrial blood residence time for non-Newtonian different constitutive laws. Panels of this Figure are presented in the same format as Figure 6, but including Newtonian results in the left column. A) Spatial distribution of residence time. B) Time histories of residence time inside the LA body and the LAA. C) Violin plots of the probability density function inside the LAA.

As expected, the thixotropic non-Newtonian simulations (CY- $\left.T_{R}-37, \mathrm{CY}-T_{R}-55\right)$ predicted lower LAA residence time values than their equilibrium counterparts (CY-37, CY-55), albeit still larger than the Newtonian simulations. However, the situation was different in the LA body, where all non-Newtonian and Newtonian simulations yielded similar results for $T_{R}$. Although, this result might seem counter-intuitive, below we argue that it is the direct consequence of residence time being dictated by mass conservation in the LA body (see Discussion section).

To quantify how non-Newtonian rheology influenced blood stasis estimations, we focused on the distal LAA, the region with highest residence time where where thrombi are most likely to form. Specifically, we considered the $33 \%$ voxels inside the LAA most distant from the ostium and computed the percentage of those occupied by stagnant blood, defined as $T_{R}$ larger than several cutoff values $(6,8$, and $10 \mathrm{~s})$. Consistent with Figure $7 \mathrm{~A}$, the fraction of the distal LAA predicted to be stagnant increased notably when including non-Newtonian effects. Table 2 shows that, when comparing the low- $H c t$ model CY-37 with the reference Newtonian simulations, the stagnant blood volume in the distal LAA increased from $8 \%$ to $24 \%$ on average for healthy subjects and from $22 \%$ to $25 \%$ for AF patients for moderate cutoff values (6 s), whereas it increased from $0 \%$ to $5.3 \%$ in healthy subjects and from $2.6 \%$ to $11 \%$ in AF patients for high cutoff values (10 s). Likewise, the maximum residence increased from 8.5 to $12 \mathrm{~s}$ on average for healthy subjects and from 10 to $14 \mathrm{~s}$ for AF patients. In addition, both the stagnant distal LAA volume and the maximum residence time increased with $H c t$ for a given given constitutive law (i.e., CY-37 with CY-55 and CY- $T_{R}-37$ with CY- $T_{R}-55$ ). Overall, these results suggest that neglecting non-Newtonian rheology can lead to underestimating the size of stagnant regions inside the LAA as well as their level of stasis. 

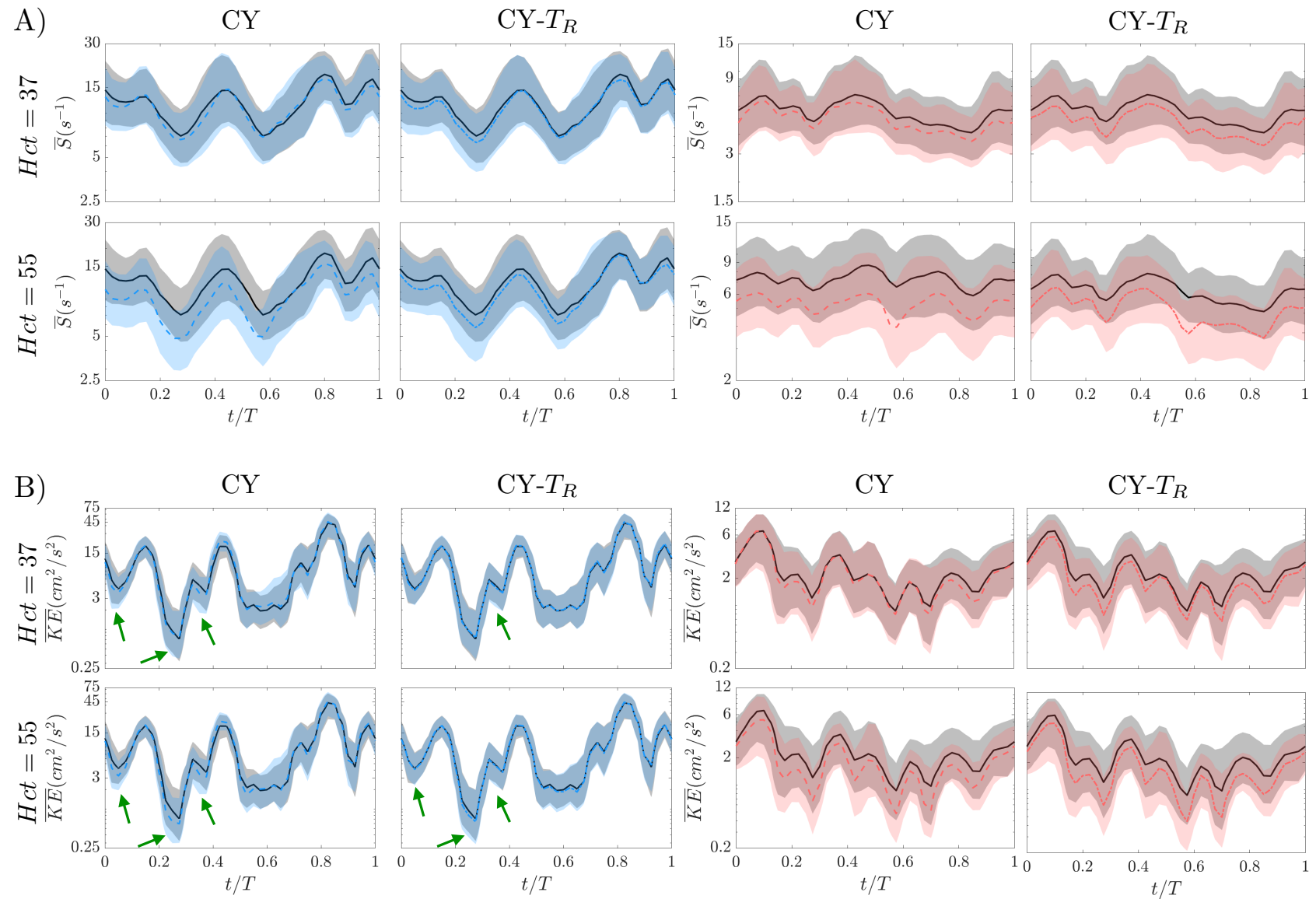

Figure 8: Shear rate and Kinetic energy in the distal LAA for Newtonian and non-Newtonian different constitutive laws. A) Time histories during one cardiac cycle of shear rate median and $25^{t h}-75^{t h}$ percentiles (denoted by colored shaded regions). The subject with normal atrial function (case 2) is represented with blue lines and patches, and the subject with an LAA thrombus and abnormal atrial function (case 5) is depicted in red. The four non-Newtonian constitutive laws are included for each case: Hct changes between rows and the constitutive models between columns. Median and $25^{t h}-75^{t h}$ percentiles of Newtonian case for the same patient are also included in black for comparison. B) Time histories during one cardiac cycle of kinetic energy median and $25^{t h}-75^{t h}$ percentiles. Panels are presented in the same format than in A).

Two other metrics commonly used in CFD analyses to summarize cardiac hemodynamics and, in particular, stasis are the flow mean shear rate $S$ and the kinetic energy, $\mathrm{KE}=(\vec{u} \cdot \vec{u}) / 2$, respectively. Figure 8 displays the median together with the $25^{t h}$ and $75^{t h}$ percentiles of these two variables inside the LAA. The data are phase-averaged for the last three cycles of the simulations and dislayed vs. time for heartbeat. The data come from the same normal subject (case 2) and AF patient with an LAA thrombus (case 5) considered in the previous figures. Simulations results from the constitutive relations CY-37, CY-55, CY- $T_{R}-37$, and CY- $T_{R}-55$ are plotted together with those from the reference Newtonian simulations (grey). In both subjects, non-Newtonian effects moderately shifted the distributions of $S$ and KE towards lower values of these variables. In the healthy subject, the shift was most pronounced during the atrial diastole and LV relaxation, while it was barely detectable during atrial systole (being less noticeable in KE, see green arrows). In the AF patient, the shift towards lower $S$ and KE was sustained throughout the whole cardiac cycle. The contrast between the large increase of $T_{R}$ and the moderate differences in $S$ or KE supports the idea that velocity changes accumulating over multiple heartbeats significantly impact residence time and blood stasis.

\section{Discussion}

The left atrial appendage (LAA) is regarded as the most likely thrombosis site in patients with AF who suffer embolic strokes $[11,12]$. We hypothesized that sustained low shear leading to increased blood viscosity could cause hemodynamics alterations and magnify LAA blood stasis [25, 26], a critical factor in thrombogenesis [45]. This study aimed to evaluate how non-Newtonian rheology could affect left atrial (LA) hemodynamics and CFD-derived metrics of LAA blood stasis, particularly residence time. Since Shettigar et al. [46] reported blood residence time to examine the washout of artificial ventricles, this quantity has been used to study the risk of intracardiac thrombosis [12, 26, 47]. Patient-specific 
bioRxiv preprint doi: https://doi.org/10.1101/2021.06.24.449801; this version posted September 9, 2021. The copyright holder for this

preprint (which was not certified by peer review) is the author/funder, who has granted bioRxiv a license to display the preprint in perpetuity. It is made available under aCC-BY-NC-ND 4.0 International license.

computational fluid dynamics (CFD) analyses are emerging as a tool to help assess LAA thrombosis risk [21]. However, previous investigations have considered constant fluid viscosity, neglecting the non-Newtonian rheology of blood at low shear rates.

\subsection{Red blood cell aggregation in the LA and LAA}

Red blood cell (RBC) aggregation into rouleaux is a significant cause of non-Newtonian blood rheology at low shear rates and long residence times. There is ample clinical evidence of RBC aggregation in the left atrium because RBC rouleaux increase blood's echogenicity creating spontaneous contrast (a.k.a. "smoke") which are readily detected in transesophageal ultrasound images [27]. Multiple studies have established a relationship between LAA blood stasis, echocardiographic spontaneous contrast, and the risk of LAA thrombosis, especially in patients with AF [45, 28]. Furthermore, previous CFD analyses of LA hemodynamics consistently report shear rates below $70 \mathrm{~s}^{-1}$ and residence times above $5 \mathrm{~s}$ inside the LAA [25, 26, 48], which, according to data from in vitro experiments, should cause RBC aggregation. However, there is a lack of studies evaluating the importance of non-Newtonian blood rheology in the LA and LAA hemodynamics. Recently, Wang et al. [49] incorporated non-Newtonian effects in their CFD simulations of blood flow of a patient-specific LA model. Yet, these authors did not specifically focus on non-Newtonian effects; instead, they investigated how beating rhythm can affect the risk of thrombosis.

\subsection{The range of Non-Newtonian effects: impact of hematocrit and rouleaux for- mation kinetics}

We considered constitutive relations based on the Carreau-Yasuda model (eq. 3), which has been extensively used in previous CFD analyses of cardiovascular flows outside of the LA [50, 51, 37]. The Carreau Yasuda model depends on several parameters that are sensitive to the hematocrit level. Hematocrit, is a major determinant of blood viscosity together with flow shear rate [22]. Normal hematocrit levels can vary among individuals within the range $H c t=[34,54]$ depending on factors like age and sex [41,42]. Thus, to evaluate the extent of potential non-Newtonian effects, we parameterized the Carreau-Yasuda model using values from the literature corresponding to Hct $=37$ and $H c t=55$ $[38,39]$. These simulations, denoted CY-37 and CY-55, allowed us to probe non-Newtonian effects for the lower and upper limits of the normal Hct range. They yielded increased blood viscosity inside the LA chamber as compared to the Newtonian simulations. The differences were especially relevant inside the LAA, where the normalized average viscosity (i.e., $\left.\langle\nu(\vec{x})\rangle / \nu_{\infty}\right)$ ranged between [1.18-1.38] for Hct $=37$ and [1.99-2.56] for Hct $=55$. These viscosity amplifications are consistent with those reported in CFD analyses of other vessels and cardiac chambers [37, 51].

The Carreau-Yasuda model presumes that rouleaux formation exclusively depends on shear rate, which is a reasonable assumption for quasi-steady flows in small vessels with simple tubular geometries (e.g., the microcirculation). In these geometries, the flow timescales are longer than those of RBC aggregation kinetics, and low shear rate implies flow separation and long residence time. However, these assumptions break down for the complex, unsteady flows observed in the cardiac chambers [52]. In vitro investigations of rouleaux formation kinetics have reported $\mathrm{RBC}$ aggregation times between 3 and $15 \mathrm{~s}$ for normal blood and between 0.5 and $1.5 \mathrm{~s}$ for pathologically hypercoagulable blood [43, 44]. To consider the interplay between these timescales and the flow timescales induced by cardac pulsatility, we ran simulations with a thixotropic constitutive relation. Specifically, we implemented a modified constitutive relation that progressively activates the Carreau-Yasuda model as residence time increases beyond $T_{\mu}=3 \mathrm{~s}$ (eqs. 4-5) [23]. We considered hematocrit levels $H c t=37$ and $H c t=55$, and denoted the corresponding simulations CY- $T_{R}-37$ and CY- $T_{R}-55$. These simulations produced viscosities close to the Newtonian reference value inside the LA body, which is replenished every 1-2 heartbeats. However, the LAA remained a site of significant non-Newtonian rheology, showing normalized average viscosities of up to $[\overline{\nu(\vec{x}, t)}]_{m a x} / \nu_{\infty}=1.07$ and 2.15 in the CY- $T_{R}-37$ and CY- $T_{R}-55$ cases considered, respectively. Our observations in the LAA contrast with the finding that considering the kinetics of rouleaux formation notably decreased shear-thinning effects in abdominal aortic and intracranial aneurysms [23]. We attribute this difference to the fact that blood residence time inside the LAA seems to be considerably longer than in aneurysms [23, 53].

\subsection{Effects of non-Newtonian rheology on LA and LAA hemodynamics and blood residence time}

There is scarce information about the influence of blood's shear-thinning rheology on intracardiac hemodynamics. In the LV, clinical observation of spontaneous echocardiographic contrast suggest RBC aggregates can form in this chamber [27], and two modeling studies have evaluated non-Newtonian effects. Doost et al. used CFD analysis to evaluate various constitutive relations on a patient-specific LV anatomical model, finding that blood viscosity increased enormously in the apical region [51]. More recently, Riva et al. analyzed patient-specific 4-D flow MRI and Hct measurements, reporting significant deviations from Newtonian behavior in areas of recirculating or low velocity [54]. It should be noted that Doost et al.'s constitutive models correspond to high hematocrit (e.g., their Carreau model is equivalent to the present 
CY-55 model). Moreover, both Doost et al. and Riva et al. neglected the kinetics of rouleaux formation. Yet, blood flow tends to be slowest and residence time tends to be highest near the LV apex [55], and these features are exacerbated after acute myocardial infarction [12]. Overall, the existing data suggest that non-Newtonian effects in the LV might be appreciable in some pathological conditions.

The influence of blood's shear-thinning on atrial hemodynamics is even more understudied than in the LV; we are unaware of previous flow imaging studies, and we are only aware of the beforementioned CFD study of Wang et al. [49]. Inside the atrial body, our Newtonian and non-Newtonian simulations produced similar flow patterns for all the constitutive models considered, even if viscosity significantly increased throughout the whole chamber for the CY-55 model. Likewise, $T_{R}$ inside the LA body was not appreciably affected by blood's shear thinning. One can explain this behavior by considering that the main flow pattern in the LA body consists of approximately straight motion from the PVs to the mitral annulus. This flow pattern is mainly dictated by global mass conservation in our simulations, whose boundary conditions drive a fluid volume equal to the LV stroke volume (LVSV) through the LA body every heartbeat. Consequently, the mean $T_{R}$ inside the LA body could be roughly approximated as $T_{R, L A \text {-body }} \approx L A V /(\alpha L V S V) \mathrm{s}$, where LAV is the mean LA volume and the factor $\alpha>1$ in the denominator accounts for most of the transport occurring during the $\mathrm{E}$ wave, which only spans a fraction of the cycle. While this estimation neglects relevant details such as the E/A ratio or the LA's reservoir and booster functions, Figure SI 1 indicates that it is a good approximation for $\alpha \approx 2$, supporting that $T_{R}$ in the LA body is indeed dictated by global chamber mass conservation, at least in our simulations. Thus, since the LA volume, LVSV, and E/A ratio were the same in our Newtonian and non-Newtonian simulations, the overall distribution of $T_{R}$ in the atrial body was not significantly altered by implementing different constitutive laws.

In contrast to the LA body, the LAA is a closed chamber excluded from the atrium's blood transit conduit that needs multiple heartbeats to be cleared and replenished. The filling and drainage jets driven by the expansion and contraction of the LAA walls play an important role in recycling the LAA's blood pool. The net flow rate these jets create through the LAA's orifice is dictated by the rate of change of LAA volume and should be relatively insensitive to variations in blood viscosity. However, we and other have demonstrated the existence of significant secondary swirling flows inside the LAA $[26,56]$, which can also transport blood into and out of the LAA even if their associated net flux is zero. In fact, we previously showed that fixed-wall simulations and moving-wall simulations produce comparable values of blood residence time inside LAAs of the same geometry [26]. Furthermore, while previous CFD analyses indicate that LAA residence time correlates inversely with the LAA ejection fraction [26], this correlation is imperfect and additional factors such as LA volume, LAA morphology, PV flow profiles, and MV have been shown to vortical patterns and residence time inside the LAA [57, 58]. Of note, LAA morphology is a well-known stroke risk factor in patients with AF [11]. Our non-Newtonian simulations suggest that the blood shear thinning not only can affect the shape of the filling and draining jets but also dampen the secondary swirling motions inside the whole appendage. These differences translate into larger values of LAA residence time when non-Newtonian blood rheology is considered, especially in the distal region near the LAA apex.

Considering different constitutive laws and hematocrit values proved helpful to evaluate the influence of non-Newtonian effects in LAA residence time. In the cases studied, the time-averaged LAA $T_{R}$ increased by up to $33 \%$, and and the maximum value of $T_{R}$ increased by up to $66 \%$. Furthermore, significantly larger volumes within the LAA presented high values of $T_{R}$ when non-Newtonian effects were considered (e.g., the volume with $T_{R}>6$ s increased by up to 14-fold). Overall, our CFD analyses suggest that non-Newtonian blood rheology significantly impacts the predicted values of LAA residence time, even for hematocrit within the normal range. Moreover, our data suggest that LAA blood stasis is exacerbated by high hematocrit, consistent with clinically observed associations between elevated hematocrit and ischemic stroke risk, especially in males and the aged [59,60]. Thus, while the current paradigm to explain this association focuses exclusively on alterations of cerebral blood flow [29], the present study implies that elevated hematocrit could also accentuate the risk of cardiac thromboembolism. Further studies are warranted to explore this potentially new mechanism.

\subsection{Study Limitations}

As mentioned above, the size of our subject cohort was too small $(N=6)$ to statistically infer or discard potential correlations between non-Newtonian effects and LA/LAA function or geometry. Nevertheless, the cohort included subjects with normal LA function and a variety of LA dysfunctions (e.g., reservoir and/or booster pump) as well as subjects in sinus rhythm and with AF. Thus, our analyses demonstrate the potential impact of non-Newtonian blood rheology in LA hemodynamics and LAA residence time for a wide range of patient-specific settings.

We did not input patient-specific values of heart rate in our simulations. Instead, we ran all our cases at 60 beats per minute, similar to our previous constant viscosity simulations [26], which we used as the Newtonian baseline in the present study. Heart rate is known to influence both shear rate and residence time in the left ventricle [61, 62], and similar dependencies are anticipated in the LA. Future studies shall address whether these phenomena cause non-Newtonian effects in the LAA to be heart-rate dependent. We did not consider patient-specific hematocrit values. Instead, we ran simulations for two hematocrit values near the lower and upper limits of this parameter's normal range to assess possible non-Newtonian effects. Likewise, the characteristic time $\left(T_{\mu}\right)$ in our thixotropic constitutive laws was not patient-specific but equal to 3 seconds for all subjects based on human RBC aggregation times measured in vitro by different techniques 
$[44,43]$.

We implemented patient-specific inflow/outflow boundary conditions, with the caveat that the PV flow rates were evenly distributed to match the boundary conditions used in Garcia-Villalba et al.'s [26] Newtonian baseline simulations. We note that, while it is possible to derive a 0D model of the pulmonary circulation to determine each PV's split of the total PV flow rate, fixing the value of the model impedances would be equivalent to directly specifying the flow split. The actual flow split is patient-specific and, given that the right lung has one more lobe than the left lung, the right PVs typically discharge more flow into the LA than the left PVs [20]. However, Lantz et al. [20] reported that flow velocities from simulations with evenly split PV flow rates agree reasonably well with patient-specific phase-contrast MRI data. Finally, we note that we did not model the geometry or motion of the mitral valve leaflets, based on previous evidence that they do not affect LA hemodynamics [15].

Our simulations are driven by mass flux boundary conditions so that a fluid volume equal to the patient-specific LVSV transits the atrium every heartbeat. This choice implies that the external work performed by the LA varies when the blood's constitutive relation is varied for each subject. However, the pressure drop between the PVs and the MV changes less than $0.3 \mathrm{mmHg}$ between our Newtonian and non-Newtonian simulations, which is negligible compared to the total pressure in the LA $(10 \mathrm{mmHg}$, see $[63,64,65]$.

\section{Conclusions}

Patient-specific CFD analyses of left atrial hemodynamics suggest that the thixotropic, shear-thinning rheology of blood can significantly affect flow patterns inside the atrial appendage in a hematocrit-dependent manner. These nonNewtonian effects, which were neglected in previous CFD studies, aggravate blood stasis and increase the likelihood of left atrial appendage thrombosis, a recognized risk factor in ischemic stroke. Our simulations open a new possibility to untangle the contribution of hematocrit to stroke risk.

Acknowledgements. This work was supported by grants from the American Heart Association (20POST35200401), the UCSD Galvanizing Engineering and Medicine Program, the National Institutes of Health (1R01HL160024), the Spanish Agency of Research (PID2019-107279RB-I00 and AEI/10.13039/501100011033), and the Regional Government of Madrid, Spain (Y2018/BIO-4858 PREFI-CM). A reciprocal visiting chair funded by the UC3M-Santander Foundation and computational time provided by XSEDE (Comet) and RES (Altamira and Caléndula) are gratefully acknowledged. Scanning electron microscopic images of RBC rouleaux (https://phil.cdc.gov/Details.aspx?pid=10902) used in the Graphical Table of Contents is also gratefully acknowledge. 


\section{Supplementary information (SI)}

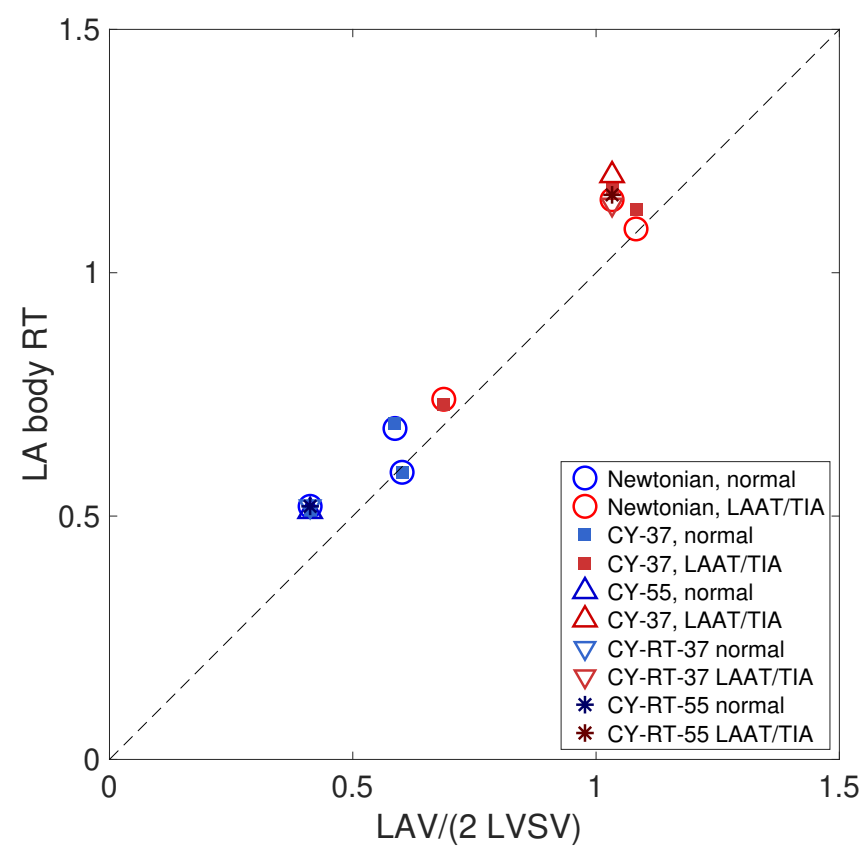

Figure SI 1: Model to predict $T_{R}$ inside of the LA body. Residence time $\left(T_{R}\right)$ approximation based on the mean LA volume (LAV), the LV stroke volume (LVSV), and a factor $\alpha$ that accounts for the fraction of the cycle the E wave spans. Symbols identify the average value obtained for subjects with normal LA function (normal) cases and subjects with LAA thrombus or a history of TIAs (LAAT/TIA) with each viscosity model used (see Table 2).

\section{References}

[1] S. S. Virani, A. Alonso, E. J. Benjamin, M. S. Bittencourt, C. W. Callaway, A. P. Carson, A. M. Chamberlain, A. R. Chang, S. Cheng, F. N. Delling, et al. Heart disease and stroke statistics - 2020 update: a report from the american heart association. Circulation, 141(9):e139-e596, 2020.

[2] E. J. Benjamin, P. Muntner, A. Alonso, M. S. Bittencourt, C. W. Callaway, A. P. Carson, A. M. Chamberlain, A. R. Chang, S. Cheng, S. R. Das, et al. Heart disease and stroke statistics - 2019 update: a report from the american heart association. Circulation, 139(10):e56-e528, 2019.

[3] R. G. Hart, H.-C. Diener, and S. J. Connolly. Embolic strokes of undetermined source: support for a new clinical construct-authors' reply. Lancet Neurol, 13(10):967, 2014.

[4] H. Kamel, P. M. Okin, M. SV Elkind, and C. Iadecola. Atrial fibrillation and mechanisms of stroke: time for a new model. Stroke, 47(3):895-900, 2016.

[5] C. A. Aakre, C. J. McLeod, S. S. Cha, T. SM Tsang, G. YH Lip, and B. J. Gersh. Comparison of clinical risk stratification for predicting stroke and thromboembolism in atrial fibrillation. Stroke, 45(2):426-431, 2014.

[6] T. J. Siddiqi, M. S. Usman, I. Shahid, J. Ahmed, S. U. Khan, L. Ya'qoub, C. S. Rihal, and M. Alkhouli. Utility of the cha2ds2-vasc score for predicting ischaemic stroke in patients with or without atrial fibrillation: a systematic review and meta-analysis. Eur J Prev Cardiol, 2021.

[7] R. G. Hart, M. Sharma, H. Mundl, S. E. Kasner, S. I. Bangdiwala, S. D. Berkowitz, B. Swaminathan, P. Lavados, Y. Wang, Y. Wang, et al. Rivaroxaban for stroke prevention after embolic stroke of undetermined source. $N$ Engl J Med, 378(23):2191-2201, 2018.

[8] W. Y. Ding, D. Gupta, and G. YH Lip. Atrial fibrillation and the prothrombotic state: revisiting virchow's triad in 2020. Heart, 106(19):1463-1468, 2020.

[9] G. Ernst, C. Stöllberger, F. Abzieher, W. Veit-Dirscherl, E. Bonner, B. Bibus, B. Schneider, and J. Slany. Morphology of the left atrial appendage. Anat Rec, 242(4):553-561, 1995.

[10] NM Al-Saady, OA Obel, and AJ Camm. Left atrial appendage: structure, function, and role in thromboembolism. Heart, 82(5):547-554, 1999.

[11] L. Di Biase, P. Santangeli, M. Anselmino, P. Mohanty, I. Salvetti, S. Gili, R. Horton, J. E. Sanchez, R. Bai, S. Mohanty, et al. Does the left atrial appendage morphology correlate with the risk of stroke in patients with atrial fibrillation? results from a multicenter study. J. Am. Coll. Cardiol, 60(6):531-538, 2012. 
bioRxiv preprint doi: https://doi.org/10.1101/2021.06.24.449801; this version posted September 9, 2021. The copyright holder for this

preprint (which was not certified by peer review) is the author/funder, who has granted bioRxiv a license to display the preprint in perpetuity. It is made available under aCC-BY-NC-ND 4.0 International license.

[12] P. Martinez-Legazpi, L. Rossini, C. Pérez del Villar, Y. Benito, C. Devesa-Cordero, R. Yotti, A. Delgado-Montero, A. Gonzalez-Mansilla, A. M. Kahn, F. Fernandez-Avilés, et al. Stasis mapping using ultrasound: a prospective study in acute myocardial infarction. JACC Cardiovasc. Imaging, 11(3):514-515, 2018.

[13] V. Mihalef, R. I. Ionasec, P. Sharma, B. Georgescu, I. Voigt, M. Suehling, and D. Comaniciu. Patient-specific modelling of whole heart anatomy, dynamics and haemodynamics from four-dimensional cardiac ct images. Interface Focus, 1(3):286-296, 2011.

[14] C. Chnafa, S. Mendez, and F. Nicoud. Image-based large-eddy simulation in a realistic left heart. Comput Fluids, 94:173-187, 2014.

[15] V. Vedula. Image based computational modeling of intracardiac flows. PhD thesis, Johns Hopkins University, 2015.

[16] V. Vedula, R. George, L. Younes, and R. Mittal. Hemodynamics in the left atrium and its effect on ventricular flow patterns. J Biomech Eng, 137(11), 2015.

[17] N. R. Saber, AD Gosman, N. B. Wood, P. J. Kilner, C. L. Charrier, and D. N. Firmin. Computational flow modeling of the left ventricle based on in vivo mri data: initial experience. Ann Biomed Eng, 29(4):275-283, 2001.

[18] Q. Long, R. Merrifield, XY Xu, P. Kilner, DN Firmin, and GZ Yang. Subject-specific computational simulation of left ventricular flow based on magnetic resonance imaging. Proc Inst Mech Eng H, 222(4):475-485, 2008.

[19] T. Schenkel, M. Malve, M. Reik, M. Markl, B. Jung, and H. Oertel. Mri-based cfd analysis of flow in a human left ventricle: methodology and application to a healthy heart. Ann Biomed Eng, 37(3):503-515, 2009.

[20] J. Lantz, V. Gupta, L. Henriksson, M. Karlsson, A. Persson, C.-J. Carlhäll, and T. Ebbers. Impact of pulmonary venous inflow on cardiac flow simulations: comparison with in vivo 4d flow mri. Ann Biomed Eng, 47(2):413-424, 2019 .

[21] P. M. Boyle, J. C. del Álamo, and N. Akoum. Fibrosis, atrial fibrillation and stroke: clinical updates and emerging mechanistic models. Heart, 107(2):99-105, 2021.

[22] S. Chien, S. Usami, R. J. Dellenback, M. I. Gregersen, L. B. Nanninga, and M. M. Guest. Blood viscosity: influence of erythrocyte aggregation. Science, 157(3790):829-831, 1967.

[23] A. Arzani. Accounting for residence-time in blood rheology models: do we really need non-newtonian blood flow modelling in large arteries? J R Soc Interface, 15(146):20180486, 2018.

[24] J. Lantz, V. Gupta, L. Henriksson, M. Karlsson, A. Persson, C.-J. Carlhäll, and T. Ebbers. Intracardiac flow at 4d ct: comparison with 4d flow mri. Radiology, 289(1):51-58, 2018.

[25] G. M. Bosi, A. Cook, R. Rai, L. J. Menezes, S. Schievano, R. Torii, and G. B. Burriesci. Computational fluid dynamic analysis of the left atrial appendage to predict thrombosis risk. Front Cardiovasc Med, 5:34, 2018.

[26] M. García-Villalba, L. Rossini, A. Gonzalo, D. Vigneault, P. Martinez-Legazpi, E. Durán, O. Flores, J. Bermejo, E. McVeigh, A. M. Kahn, et al. Demonstration of patient-specific simulations to assess left atrial appendage thrombogenesis risk. Front Physiol, 12, 2021.

[27] A. Merino, P. Hauptman, L. Badimon, J. J. Badimon, M. Cohen, V. Fuster, and M. Goldman. Echocardiographic "smoke" is produced by an interaction of erythrocytes and plasma proteins modulated by shear forces. J. Am. Coll. Cardiol, 20(7):1661-1668, 1992.

[28] I. W. Black. Spontaneous echo contrast: where there's smoke there's fire. Echocardiography, 17(4):373-382, 2000.

[29] K. Stavropoulos, K. P. Imprialos, S. Bouloukou, C. Boutari, and M. Doumas. Hematocrit and stroke: a forgotten and neglected link? In Seminars in thrombosis and hemostasis, volume 43, pages 591-598, 2017.

[30] P. A. Yushkevich, J. Piven, H. C. Hazlett, R. G. Smith, S. Ho, J. C. Gee, and G. Gerig. User-guided 3d active contour segmentation of anatomical structures: significantly improved efficiency and reliability. Neuroimage, 31(3):1116$1128,2006$.

[31] Q. Fang and D. A. Boas. Tetrahedral mesh generation from volumetric binary and grayscale images. In 2009 IEEE International Symposium on Biomedical Imaging: From Nano to Macro, pages 1142-1145, 2009.

[32] A. Myronenko and X. Song. Point set registration: Coherent point drift. IEEE PAMI, 32(12):2262-2275, 2010.

[33] M. Moriche, O. Flores, and M. García-Villalba. On the aerodynamic forces on heaving and pitching airfoils at low Reynolds number. J. Fluid Mech., 828:395-423, 2017.

[34] G. P. Galdi, R. Rannacher, A. M. Robertson, and S. Turek. Hemodynamical flows. Delhi Book Store, 2008.

[35] L. Rossini, P. Martinez-Legazpi, V. Vu, L. Fernandez-Friera, C. Pérez del Villar, S. Rodriguez-Lopez, Y. Benito, M.-G. Borja, D. Pastor-Escuredo, R. Yotti, et al. A clinical method for mapping and quantifying blood stasis in the left ventricle. J. Biomech, 49(11):2152-2161, 2016.

[36] C.-W. Shu. Essentially non-oscillatory and weighted essentially non-oscillatory schemes for hyperbolic conservation laws. In Advanced numerical approximation of nonlinear hyperbolic equations, pages 325-432. Springer, 1998.

[37] F. De Vita, MD De Tullio, and R. Verzicco. Numerical simulation of the non-newtonian blood flow through a mechanical aortic valve. Theor Comput Fluid Dyn, 30(1-2):129-138, 2016.

[38] A. Leuprecht and K. Perktold. Computer simulation of non-newtonian effects on blood flow in large arteries. Comput Methods Biomech Biomed Engin, 4(2):149-163, 2001. 
bioRxiv preprint doi: https://doi.org/10.1101/2021.06.24.449801; this version posted September $9,2021$. The copyright holder for this

preprint (which was not certified by peer review) is the author/funder, who has granted bioRxiv a license to display the preprint in perpetuity. It is made available under aCC-BY-NC-ND 4.0 International license.

[39] M. G. Al-Azawy, A. Turan, and A. Revell. Investigating the impact of non-newtonian blood models within a heart pump. Int J Numer Method Biomed Eng, 33(1):e02780, 2017.

[40] S. Chien, S. Usami, H. M. Taylor, J. L. Lundberg, and M. I. Gregersen. Effects of hematocrit and plasma proteins on human blood rheology at low shear rates. J. Appl. Physiol, 21(1):81-87, 1966.

[41] B. Devine. Mean blood hematocrit of adults: United States, 1960-1962. Number 19-24. US Department of Health, Education, and Welfare, Public Health Service, 1967.

[42] H. H. Billett. Hemoglobin and hematocrit. Clinical Methods: The History, Physical, and Laboratory Examinations. 3rd edition, 1990.

[43] CR Huang and W. Fabisiak. Thixotropic parameters of whole human blood. Thromb. Res, 8:1-8, 1976.

[44] H. Schmid-Schönbein, E. Volger, and HJ Klose. Microrheology and light transmission of blood. Pflügers Archiv, 333(2):140-155, 1972.

[45] D. Fatkin, R. P. Kelly, and M. P. Feneley. Relations between left atrial appendage blood flow velocity, spontaneous echocardiographic contrast and thromboembolic risk in vivo. J. Am. Coll. Cardiol, 23(4):961-969, 1994.

[46] U. R. Shettigar, M. Dropmann, P. E. Christian, and W. J. Kolff. Residence time distributions in artificial ventricles. ASAIO Trans, 35(3):708-712, 1989.

[47] J. H. Seo and R. Mittal. Effect of diastolic flow patterns on the function of the left ventricle. Phys. Fluids, 25(11):110801, 2013.

[48] S. Sanatkhani, S. Nedios, P. G. Menon, A. Bollmann, G. Hindricks, and S. G. Shroff. Subject-specific calculation of left atrial appendage blood-borne particle residence time distribution in atrial fibrillation. Front Physiol, $12,2021$.

[49] Y. Wang, Y. Qiao, Y. Mao, C. Jiang, J. Fan, and K. Luo. Numerical prediction of thrombosis risk in left atrium under atrial fibrillation. Math Biosci Eng, 17(3):2348-2360, 2020.

[50] J. Biasetti, T. C. Gasser, M. Auer, U. Hedin, and F. Labruto. Hemodynamics of the normal aorta compared to fusiform and saccular abdominal aortic aneurysms with emphasis on a potential thrombus formation mechanism. Ann Biomed Eng, 38(2):380-390, 2010.

[51] S. N. Doost, L. Zhong, B. Su, and Y. S. Morsi. The numerical analysis of non-newtonian blood flow in human patient-specific left ventricle. Comput Methods Programs Biomed, 127:232-247, 2016.

[52] S. Hendabadi, J. Bermejo, Y. Benito, R. Yotti, F. Fernández-Avilés, J. C. del Álamo, and S. C. Shadden. Topology of blood transport in the human left ventricle by novel processing of doppler echocardiography. Ann Biomed Eng, 41(12):2603-2616, 2013

[53] G.-Y. Suh, A. S. Les, A. S. Tenforde, S. C. Shadden, R. L. Spilker, J. J. Yeung, C. P. Cheng, R. J. Herfkens, R. L. Dalman, and C. A. Taylor. Quantification of particle residence time in abdominal aortic aneurysms using magnetic resonance imaging and computational fluid dynamics. Ann Biomed Eng, 39(2):864-883, 2011.

[54] A. Riva, F. Sturla, A. Caimi, S. Pica, D. Giese, P. Milani, G. Palladini, M. Lombardi, A. Redaelli, and E. Votta. 4d flow evaluation of blood non-newtonian behavior in left ventricle flow analysis. J. Biomech., 119:110308, 2021.

[55] Y. Benito, P. Martinez-Legazpi, L. Rossini, C. Pérez del Villar, R. Yotti, Y. Martin Peinador, D. Rodriguez-Perez, M. Desco, C. Medrano, J. C. Antoranz, et al. Age-dependence of flow homeostasis in the left ventricle. Front Physiol, 10:485, 2019.

[56] A. Masci, L. Barone, L. Dedè, M. Fedele, C. Tomasi, A. Quarteroni, and C. Corsi. The impact of left atrium appendage morphology on stroke risk assessment in atrial fibrillation: a computational fluid dynamics study. Front Physiol, 9:1938, 2019.

[57] G. García-Isla, A. L. Olivares, E. Silva, M. Nuñez-Garcia, C. Butakoff, D. Sanchez-Quintana, H. G. Morales, X. Freixa, J. Noailly, T. De Potter, et al. Sensitivity analysis of geometrical parameters to study haemodynamics and thrombus formation in the left atrial appendage. Int J Numer Method Biomed Eng, 34(8):e3100, 2018.

[58] E. Duran, M. García-Villalba, L. Rossini, A. Gonzalo, D. Vigneault, P. Martinez-Legazpi, J. Bermejo, E. McVeigh, A. M. Kahn, J. C. del Álamo, et al. Effect of pulmonary vein inflow on patient-specific cfd prediction of left atrial blood stasis. Bull. Am. Phys. Soc, 2020.

[59] H. Tohgi, H. Yamanouchi, M. Murakami, and M. Kameyama. Importance of the hematocrit as a risk factor in cerebral infarction. Stroke, 9(4):369-374, 1978.

[60] R. Yang, A. Wang, L. Ma, Z. Su, S. Chen, Y. Wang, S. Wu, and C. Wang. Hematocrit and the incidence of stroke: a prospective, population-based cohort study. Ther Clin Risk Manag, 14:2081, 2018.

[61] V. Vasudevan, A. J. J. Low, S. P. Annamalai, S. Sampath, K. K. Poh, T. Totman, M. Mazlan, G. Croft, A. M. Richards, D. PV de Kleijn, et al. Flow dynamics and energy efficiency of flow in the left ventricle during myocardial infarction. Biomech. Model. Mechanobiol, 16(5):1503-1517, 2017.

[62] L. Rossini, P. Martinez-Legazpi, Y. Benito, C. Pérez del Villar, A. Gonzalez-Mansilla, A. Barrio, M.-G. Borja, R. Yotti, A. M. Kahn, S. C. Shadden, et al. Clinical assessment of intraventricular blood transport in patients undergoing cardiac resynchronization therapy. Meccanica, 52(3):563-576, 2017.

[63] J. Ross Jr., E. Braunwald, and A. G. Morrow. Transseptal left atrial puncture: new technique for the measurement of left atrial pressure in man. Am. J. Cardiol, 3(5):653-655, 1959. 
[64] C. Stefanadis, J. Dernellis, and P. Toutouzas. A clinical appraisal of left atrial function. Eur. Heart J, 22(1):22-36, 2001.

[65] P. S Pagel, F. Kehl, M. Gare, D. A. Hettrick, J. R. Kersten, and D. C. Warltier. Mechanical function of the left atrium: new insights based on analysis of pressure-volume relations and doppler echocardiography. Anesthesiology. 